\title{
Hydrodynamic fluctuations in thermostatted multiparticle collision dynamics
}

\author{
Humberto Hijar* \\ Institute for Advanced Simulation, Jülich Supercomputing Centre, Research Centre Jülich, D-52425 Jülich, Germany and \\ Facultad de Ciencias, Universidad Nacional Autónoma de México, Circuito Exterior de Ciudad Universitaria, 04510, D. F., Mexico \\ Godehard Sutmann ${ }^{\dagger}$ \\ Institute for Advanced Simulation, Jülich Supercomputing Centre, Research Centre Jülich, D-52425 Jülich, Germany,
} (Received 22 September 2009; revised manuscript received 3 December 2010; published 20 April 2011)

\begin{abstract}
In this work we study the behavior of mesoscopic fluctuations of a fluid simulated by Multiparticle Collision Dynamics when this is applied together with a local thermostatting procedure that constrains the strength of temperature fluctuations. We consider procedures in which the thermostat interacts with the fluid at every simulation step as well as cases in which the thermostat is applied only at regular time intervals. Due to the application of the thermostat temperature fluctuations are forced to relax to equilibrium faster than they do in the nonthermostatted, constant-energy case. Depending on the interval of application of the thermostat, it is demonstrated that the thermodynamic state changes gradually from isothermal to adiabatic conditions. In order to exhibit this effect we compute from simulations diverse correlation functions of the hydrodynamic fluctuating fields. These correlation functions are compared with those predicted by a linearized hydrodynamic theory of a simple fluid in which a thermostat is applied locally. We find a good agreement between the model and the numerical results, which confirms that hydrodynamic fluctuations in Multiparticle Collision Dynamics in the presence of the thermostat have the properties expected for spontaneous fluctuations in fluids in contact with a heat reservoir.
\end{abstract}

DOI: 10.1103/PhysRevE.83.046708

PACS number(s): 47.11.-j, 05.40.-a, 02.70.Ns

\section{INTRODUCTION}

The simulation of complex fluids, e.g., dissolved chemically reacting systems, colloidal or polymer suspensions, multiphase fluids and amphiphilic systems, to mention but a few, represents a major task in computational physics. The main difficulties faced when simulations of these systems are implemented arise from the coexistence of widely separated length and timescales. Indeed, the interest in these systems lays usually in the microscopic dynamics of some degrees of freedom interacting with a solvent whose dynamics over long distances and long timescales is essential for the phenomena but whose detailed properties are irrelevant [1]. Simulating the solvent on an atomistic level by means of, e.g., molecular dynamics [2-4] on mesoscopic or even macroscopic dimensions is not feasible due to the enormous number of degrees of freedom as well as long timescales that have to be covered.

Therefore, taking into account hydrodynamic effects in particle-based simulations is a challenge that has motivated the development of new approaches to modeling hydrodynamics that incorporate essential dynamical properties and allow for coupling with the interesting microscopic degrees of freedom, yet are simple enough to be simulated for long times and distances. Among others, these methods include Brownian Dynamics $[5,6]$, in which the effect of the solvent on solutes is modeled by the Oseen- or Rotne-Prager tensor and through random displacements of the suspended particles governed by correlated multivariant Gaussian probability distribution functions; Stokesian Dynamics [7], which incorporates many-

\footnotetext{
*Also at: SNI Mexico. hijar@daad-alumni.de

${ }^{\dagger}$ g.sutmann@fz-juelich.de
}

body hydrodynamic interactions between dissolved particles through an analytical representation of the mobility tensor; and lattice Boltzmann techniques [8], which can be viewed as a special finite difference scheme for the kinetic equation of the discrete-velocity distribution function [9] and are particularly useful in fluid flow applications involving interfacial dynamics and complex boundaries $[9,10]$.

More recently, Malevanets and Kapral developed a new appealing method for simulating the dynamics of simple fluids at the mesoscopic scale $[1,11]$, which also allows for coupling with microscopic degrees of freedom. This method is known in the literature as Stochastic Rotation Dynamics or Multiparticle Collision Dynamics (MPCD) and simulates a fluid by means of particles whose positions and velocities are considered as continuous variables. The microscopic details about these particles are not specified, but their dynamics is treated in a simplified form through stochastic collisions, i.e., transformations in velocity space, which preserve both momentum and energy. Consequently, MPCD allows for recovering the hydrodynamic equations of mass and momentum conservation, and of heat conduction [1].

MPCD has some advantages that make it very attractive for the simulation of complex fluids. For instance, since MPCD is a particle-based method it can be easily coupled to suspended particles, e.g., polymers or colloids, whose detailed evolution can be followed by using molecular dynamics [11-14]. MPCD captures the hydrodynamic behavior of the fluid around the embedded particles, and, thus, it naturally simulates the hydrodynamic interactions between them [14]. In addition, due to its stochastic character it also gives rise to hydrodynamic fluctuations and, consequently, to random Brownian forces on the suspended particles $[14,15]$. In this way, MPCD can be used as a thermal bath that supports hydrodynamic 
interactions. Moreover, the algorithm of MPCD is relatively simple, which makes it very stable and suitable for simulations over large timescales. Finally, this simplified dynamics has made also possible the analytical calculation of the transport coefficients of the simulated fluid. Expressions for viscosities and thermal conductivities of MPCD fluids have been obtained from discrete-time projection operator techniques [16-18] and from a kinetic theory approach [19]. Both approaches are complementary, and simulation results have been found to agree very well with the analytical expressions, which has given confidence in using MPCD for simulating physical systems as diverse as suspensions of polymers [20] and colloids [11,14], polymers under flow [15,21,22], flow around objects $[23,24]$, and vesicles under flow [25]. Due to the extent of its present and future applications, characterizing fluids simulated via MPCD or variations of this method is a matter of significant importance.

Special boundary conditions and external force fields are usually implemented in MPCD with diverse purposes. In Ref. [26], Lees-Edwards boundary conditions [27] have been incorporated to the MPCD algorithm, in order to generate a steady shear flow. In Ref. [24], an external gravitational field and bounce-back boundary conditions in which velocities are completely reversed have been used in order to produce a steady Poiseuille flow of a MPCD fluid. In Ref. [28], the presence of the gravitational field is taken into account to study the sedimentation of claylike colloids embedded in a MPCD fluid. In these cases, work is continuously performed on the MPCD particles as they cross the boundaries and by the external force fields. Eventually, this amount of work is dissipated as heat, the temperature of the fluid increases, and it is absolutely necessary to apply a thermostatting procedure to remove the excess of energy and prevent the fluid from heating [14,24,26,28]. Although it is shown in Refs. [24,26] that the application of a thermostat is not significant in determining the shear viscosity of a MPCD fluid, it can be intuitively expected that the coupling with a thermostat will change some other physical features of this system; in particular, it may affect the way in which its thermal fluctuations are produced and propagate, e.g., sound modes should propagate at the isothermal sound speed instead of propagating at the isentropic sound speed, and temperature fluctuations will not be able to propagate through the fluid since they will be eliminated by the thermostat. As far as we know, these effects have not been addressed in the literature of the subject.

The main purpose of the present paper is to analyze the effects of a thermostat on the dynamics of the spontaneous fluctuations of an equilibrated fluid simulated with MPCD. We will examine these effects by performing measurements of the dynamic correlation functions of fluctuations in thermostatted MPCD fluids and comparing our results with analytical predictions obtained from the Fluctuating Hydrodynamics (FH) theory of Landau and Lifshitz [29] extended for liquids that interact with a local thermostat. It is worth stressing that this approach follows the recent work of Ref. [30], where the analysis of the equilibrium fluctuations of hydrodynamic modes has been used as an alternative method to directly measure transport coefficients, i.e., shear and bulk viscosities and thermal diffusivities, in MPCD fluids.

In the following section we will describe the basic implementation of MPCD and the specific thermostatting procedure to be used in this work. In order to analyze the effects of the thermostat, we will consider different strengths for its coupling with MPCD. This will be done by allowing the thermostat to interact with the system at different time rates. A strong interaction will be obtained by applying the thermostat at every simulation step, while a weaker interaction can be achieved by applying the thermostat at larger regular intervals of simulation steps. These different types of interactions between the thermostat and the fluid will be referred to as Strong Thermostatting Interaction (STI) and Partial Thermostatting Interaction (PTI), respectively. In Sec. III we will develop the FH formalism for fluids in which a thermostat is applied locally. This model will be a first approximation intended to imitate the transport of energy occurring in thermostatted MPCD. Within our model, the principal effect of the thermostat will consist of an additive contribution to the relaxation rate of temperature fluctuations. We will analyze the typical relaxation times of the hydrodynamic fluctuations in MPCD and show that the application of the thermostat might induce an effective separation of the timescales associated with relaxation of temperature and relaxation of the remaining hydrodynamic variables. We will use the formalism developed in Sec. III in order to calculate diverse dynamic correlation functions of fluctuating variables for both STI and PTI. In Sec. IV we will present measurements of these correlations obtained from direct simulations of thermostatted MPCD. We will introduce the comparison of the analytical expressions with the results of simulations and find a very good agreement. Consequently, in the present work we show that thermostatted MPCD is a useful and reliable method for simulating fluctuating hydrodynamics in isothermal conditions. As an indirect result we will also conclude that the theoretical expressions for the transport coefficients of MPCD fluids obtained previously in literature $[18,30-32]$ can be considered valid as well when these fluids are in contact with a heat reservoir. In Sec. V we will summarize our main conclusions and state some limitations of our analysis.

\section{THERMOSTATTED MULTIPARTICLE COLLISION DYNAMICS}

The MPCD algorithm simulates the dynamics of $N$ point particles of mass $m$, whose positions and velocities are specified by the vectors $\vec{r}_{i}$ and $\vec{v}_{i}$, with $i=1,2, \ldots, N$. We follow the simplest implementation of the MPCD method in which the simulation box where particles move is a cube of volume $L^{3}$. The box is subdivided into smaller cells of volume $a^{3}$. The number of particles per cell may change, but the total number of simulated particles remains constant. The simulation consists of two main steps. In the first step, also called the streaming step, particles are advanced ballistically during the time interval $\Delta t$ :

$$
\vec{r}_{i}(t+\Delta t)=\vec{r}_{i}(t)+\Delta t \vec{v}_{i}(t) .
$$


In the second step, also called the collision step, the center of mass velocity of each cell is calculated:

$$
\vec{u}_{\mu}(t)=\frac{1}{N_{\mu}} \sum_{i \in \mu} \vec{v}_{i}(t),
$$

where the xindex $\mu$ has been used to indicate the $\mu$ th cell. Then the particles located in the same cell collide and therefore exchange momentum. This collision process is produced by updating the velocities according to

$$
\vec{v}_{i}(t+\Delta t)=\vec{u}_{\mu}(t)+\mathbf{R}\left[\alpha ; \vec{n}_{\mu}(t)\right] \cdot\left[\vec{v}_{i}(t)-\vec{u}_{\mu}(t)\right],
$$

where $\mu$ indicates the cell where the $i$ th particle is located and

$$
\mathbf{R}\left(\alpha ; \vec{n}_{\mu}(t)\right)=\left(\begin{array}{lcc}
n_{\mu, x}^{2}(1-\cos \alpha)+\cos \alpha & n_{\mu, x} n_{\mu, y}(1-\cos \alpha)-n_{\mu, z} \sin \alpha & n_{\mu, x} n_{\mu, z}(1-\cos \alpha)+n_{\mu, y} \sin \alpha \\
n_{\mu, x} n_{\mu, y}(1-\cos \alpha)+n_{\mu, z} \sin \alpha & n_{\mu, y}^{2}(1-\cos \alpha)+\cos \alpha & n_{\mu, y} n_{\mu, z}(1-\cos \alpha)-n_{\mu, x} \sin \alpha \\
n_{\mu, x} n_{\mu, z}(1-\cos \alpha)-n_{\mu, y} \sin \alpha & n_{\mu, y} n_{\mu, z}(1-\cos \alpha)+n_{\mu, x} \sin \alpha & n_{\mu, z}^{2}(1-\cos \alpha)+\cos \alpha
\end{array}\right)
$$

is a stochastic rotation matrix that rotates velocities by a fixed angle $\alpha$ around the axis $\vec{n}_{\mu}$, which is chosen randomly for each collision cell in every time step. In the three-dimensional case different forms of producing the stochastic rotation exist [33]. In our simulations, the vector $\vec{n}_{\mu}$ is produced in each cell at every time step by randomly selecting a point on the surface of a sphere with unit radius.

We apply periodic boundary conditions in the three Cartesian directions. A homogeneous random displacement of the MPCD cells by a vector with components between $-a / 2$ and $a / 2$ is applied before the collision step, which guarantees Galilean invariance of the method, a fact first noticed by Ihle and Kroll [17,18].

In a MPCD liquid, hydrodynamic fields can be measured locally, i.e., on the cell level. In particular, the temperature of the $\mu$ th cell at time $t$ is determined by the mean square of the relative velocities of the particles with respect to the center of mass velocity of the cell, i.e.,

$$
T_{\mu}(t)=\frac{m}{3\left(N_{\mu}-1\right) k_{B}} \sum_{i \in \mu}\left[\vec{v}_{i}(t)-\vec{u}_{\mu}(t)\right]^{2},
$$

where $T_{\mu}$ denotes the temperature of the $\mu$ th cell, and $k_{B}$ is the Boltzmann constant.

In this expression for the temperature, it has been taken into account that the center of mass velocity of the cell does not contribute to the thermal energy on a cell level [30].

Different thermostatting procedures are known in literature for keeping the temperature of MPCD fluids at a fixed or fluctuating value around a specific temperature $[13,28]$.

In this work we consider the effect of a local thermostat, allowing for fluctuations of thermal energy on a cell level, but fixing the temperature on average to the exact prescribed value, $T_{0}$. This thermostat, called a Maxwell-Boltzmann Scaling Thermostat (MBST), was introduced and compared with other thermostats in Ref. [36]. It was shown that it preserves important statistical properties of the fluid, e.g., velocity distributions, density, and velocity profiles, to name a few. Instead of sampling a velocity scaling factor from the Maxwell-Boltzmann distribution of velocities by a Monte Carlo scheme [28,34,35], the kinetic energy is directly obtained from its thermal distribution function.
The basic principle of the thermostat is that energy fluctuations given a small number of degrees of freedom are considered. The distribution function for the kinetic energy $E_{\mu}$ on the cell level is given by

$$
\begin{aligned}
P\left(E_{\mu} \mid N_{\mu}\right)= & \frac{1}{Z} \int d^{3 N} \Delta \vec{v} \delta\left(E-\frac{m}{2} \sum_{i=1}^{N_{\mu}} \Delta \vec{v}_{i, \mu}^{2}\right) \\
& \times \delta\left(\sum_{i=1}^{N_{\mu}} \Delta \vec{v}_{i, \mu}\right) \exp \left\{-\frac{m}{2 k_{B} T} \sum_{i=1}^{N_{\mu}} \Delta \vec{v}_{i, \mu}^{2}\right\},
\end{aligned}
$$

with $N_{\mu}$ being the number of particles in a cell and $Z=$ $\int d^{3 N} \Delta \vec{v} \delta\left(\Delta \vec{v}_{i, \mu}\right) \exp \left\{-\frac{m}{2 k_{B} T} \sum_{i=1}^{N_{\mu}} \Delta \vec{v}_{i, \mu}^{2}\right\}$ the partition function. The second $\delta$ function takes into account that in the presence of an external field only relative velocities, $\Delta \vec{v}_{i, \mu}=$ $\vec{v}_{i}-\vec{u}_{\mu}$, obey the Maxwell-Boltzmann distribution. After evaluation of the integrals, the distribution function reduces to

$$
P\left(E_{\mu} \mid N_{\mu}\right)=\frac{1}{E_{\mu}}\left(\frac{E_{\mu}}{k_{B} T}\right)^{\frac{\phi}{2}} \frac{1}{\Gamma(\phi / 2)} e^{-E_{\mu} / k_{B} T},
$$

where $\phi=3\left(N_{\mu}-1\right)$ is the number of degrees of freedom within a collision cell and $\Gamma$ represents the Gamma function. According to the distribution function, Eq. (7), a random value for the cell energy $E_{\mu}^{\prime}$ is drawn appropriate for the number of particles within a cell (which is a fluctuating quantity). A random number that obeys this distribution is generated by an acceptance-rejection method (see Appendix A). In a next step, the velocities of particles within a given cell $\mu$ are scaled according to

$$
\begin{gathered}
\vec{v}_{i}(t) \rightarrow \xi_{\mu}(t)\left[\vec{v}_{i}(t)-\vec{u}_{\mu}(t)\right]+\vec{u}_{\mu}(t), \\
\xi_{\mu}=\sqrt{\frac{2 E_{\mu}^{\prime}}{m \sum_{i=1}^{N_{\mu}}\left(\vec{v}_{i}-\vec{u}_{\mu}\right)^{2}}} .
\end{gathered}
$$

This procedure guarantees conservation of momentum as well as thermal statistical properties of the fluid.

In order to study further the effects of the thermostat on the collective properties of the MPCD fluid we perform a second thermostatting implementation which we have called PTI 
previously. In this case, the velocity transformation, Eq. (8), is not implemented at every simulation step but at regular time intervals of size $n_{\text {therm }} \Delta t$, with $n_{\text {therm }}>1$.

It could be expected intuitively that due to the application of both strong and partial thermostats, the way in which temperature fluctuations evolve in the MPCD fluid is modified. Consider, for instance, the STI. Although thermal energy is exchanged between cells in the streaming step, thermal perturbations are not able to propagate through the system since they are destroyed by scaling. Because of the thermostat, temperature fluctuations effectively decay on a very short timescale, while density and velocity fluctuations are still present and evolve toward equilibrium slowly. On the other hand, considering in PTI a very large interval between applications of the thermostat, i.e., $n_{\text {therm }} \rightarrow \infty$, there will be effectively no influence on the thermal diffusion. These two extreme scenarios $\left(n_{\text {therm }}=1\right.$ and $n_{\text {therm }} \rightarrow \infty$ ) correspond to the isothermal and adiabatic conditions in the fluid, and therefore it might be expected that e.g., consequences for the dispersion relation of sound propagation might be observed. In this work we will show formally and from simulation results that this is the case. Furthermore, we will show that depending on the size of the interval $n_{\text {therm }}$ there is a gradual change from isothermal to adiabatic conditions in the observed spectra of density fluctuations, which is taken into account by an energy source function within the formalism of FH. With this purpose we will first consider from an analytic point of view the behavior of thermal hydrodynamic fluctuations occurring in simple liquids with different couplings to an energy source function.

\section{HYDRODYNAMIC FLUCTUATIONS IN THEMOSTATTED FLUIDS}

\section{A. Fluctuating hydrodynamics}

In a recent publication [30], it has been shown that the hydrodynamic fluctuations in MPCD fluids around equilibrium states can be very well described by the linearized hydrodynamics theory that is used to calculate the dynamic correlation functions of simple liquids in the limit of small wavevectors and frequencies [37,38]. In this work we will exploit this result in order to describe analytically the fluctuations occurring in a thermostatted MPCD fluid also in terms of a linearized hydrodynamic theory. In particular we will consider the FH formalism of Landau and Lifshitz [29], which can be equivalently used to describe spontaneous fluctuations in liquids. FH has been extended to calculate dynamic correlation functions of complex fluids in equilibrium and nonequilibrium stationary states [39-42]. In the present work, we will actually generalize $\mathrm{FH}$ in order to calculate correlation functions in liquids interacting periodically with a local thermostat. Although local thermostats are difficult to conceive in real systems, they can be easily implemented in a MPCD simulation, as has been described in a previous section. Our model will be an approximation resembling the exchange of energy occurring at the local level in thermostatted MPCD due to the application of the MBST.

As usual, fluctuating variables are defined as the local instantaneous deviations of the hydrodynamic fields of density $\rho$, velocity $\vec{u}$, internal energy per unit mass $e$, etc., with respect to their average values, i.e., $\delta \rho(\vec{r}, t)=\rho(\vec{r}, t)-\rho_{0}, \delta u_{i}(\vec{r}, t)=$ $u_{i}(\vec{r}, t)-u_{i, 0}, \delta e(\vec{r}, t)=e(\vec{r}, t)-e_{0}$, etc., where the subscript 0 denotes the uniform, equilibrium value of the corresponding field. In order to construct the evolution equations for the fluctuating fields, the following remarks should be considered.

First, the general hydrodynamic equations that describe the time and space evolution of the fluid are mathematical expressions for the laws of conservation of mass, momentum, and energy and therefore must be valid for describing the fluctuating motion as well [29]. In addition, for fluids in states far from a critical point, as it is the case for MPCD fluids, fluctuations can be considered small, and the general conservation equations may be linearized in terms of the fluctuating fields. Furthermore, it should be taken into account that in the presence of thermal motion, stochastic heat and momentum fluxes may appear that are not produced by temperature or velocity gradients [29]. Thus, the heat flux and and the stress tensor have fluctuating contributions that will be denoted by $Q_{i}$ and $\Sigma_{i j}$, respectively.

Finally, when the fluid is allowed to interact with a local thermostat, a source term, $\Xi=\Xi(\vec{r}, t)$, must be included in the energy balance equation, which accounts for the energy per unit time per unit volume introduced or extracted by the thermostat at position $\vec{r}$ and time $t$. For the specific case of the application of a local thermostat at regular time intervals of size $\tau$ in MPCD, we will consider, as a first approximation, that $\Xi$ can be written in the form

$$
\Xi \simeq \frac{E^{\mathrm{a}}-E^{\mathrm{b}}}{\tau a^{3}},
$$

where $E^{\mathrm{b}}=E^{\mathrm{b}}(\vec{r}, t)$ and $E^{\mathrm{a}}=E^{\mathrm{a}}(\vec{r}, t)$ are the energies at time $t$, of the cell located at position $\vec{r}$, before and after the application of the thermostat, respectively. On the one hand, $E^{\mathrm{b}}$ can be written as $E^{\mathrm{b}}=E_{0}+\rho a^{3} \delta e$, where $E_{0}$ is the average internal energy per cell. On the other hand, $E^{\mathrm{a}}$ is the energy that is assigned randomly every time interval of size $\tau$, according to the probability distribution Eq. (7). This allows us to recast our approximation, Eq. (9), as follows:

$$
\Xi \simeq \frac{\delta \mathcal{E}-\rho \delta e}{\tau},
$$

where $\delta \mathcal{E}=\left(E^{\mathrm{a}}-E_{0}\right) / a^{3}$ is the amount of energy per unit volume added or subtracted from the system by the thermostat with respect to the mean energy.

Consequently, the linearized equations describing the evolution of the fluctuating fields can be cast into the form

$$
\begin{gathered}
\partial_{t} \delta \rho+\rho_{0} \partial_{i} \delta u_{i}=0, \\
\partial_{t} \delta u_{i}+\frac{c_{T}^{2}}{\rho_{0}} \partial_{i} \delta \rho+\alpha_{T} c_{T}^{2} \partial_{i} \delta T-\frac{1}{\rho_{0}} v_{i j k l} \partial_{j} \partial_{k} \delta u_{l} \\
=-\frac{1}{\rho_{0}} \partial_{j} \Sigma_{i j}, \\
\partial_{t} \delta T-\gamma D_{T} \partial_{j} \partial_{j} \delta T+\frac{1}{\tau} \delta T+\frac{\gamma-1}{\alpha_{T}} \partial_{i} \delta u_{i} \\
=-\frac{1}{\rho_{0} c_{V}} \partial_{i} Q_{i}+\frac{1}{\tau \rho_{0} c_{V}} \delta \mathcal{E} .
\end{gathered}
$$

In these expressions $\partial_{t}$ and $\partial_{i}$ represent partial derivatives with respect to time and Cartesian coordinates, respectively, $\delta T$ represents temperature fluctuations, $c_{T}^{2}$ is the isothermal 
sound speed, $\alpha_{T}$ is the thermal expansion coefficient, $c_{V}$ is the specific heat at constant volume, $\gamma=c_{p} / c_{V}$ is the ratio of specific heats at constant pressure and volume, $D_{T}=\kappa / \rho_{0} c_{p}$ is the thermal diffusivity coefficient, and $v_{i j k l}$ is the viscous tensor:

$$
v_{i j k l}=\eta\left(\delta_{i k} \delta_{j l}+\delta_{i l} \delta_{j k}-\frac{2}{3} \delta_{i j} \delta_{k l}\right)+\zeta \delta_{i j} \delta_{k l},
$$

where $\eta$ and $\zeta$ are the shear and bulk viscosity coefficients, respectively. Notice that summation over repeated indices will be implicit throughout this paper.

Strictly speaking, the previous expression for the viscous tensor is valid for a Navier-Stokes fluid and not for a MPCD fluid for which angular momentum is not conserved [30,43]. As a consequence, the viscous tensor should have an antisymmetric contribution that modifies the sound attenuation coefficient. Later we will consider this effect explicitly, when we calculate the dynamic correlations of the fluctuations in MPCD fluids.

In order to obtain the closed form of Eqs. (11)-(13) in the representation $\{\delta \rho, \delta \vec{u}, \delta T\}$, we have used the following thermodynamic relations, which are valid for small deviations with respect to equilibrium:

$$
\begin{gathered}
\delta p=c_{T}^{2} \delta \rho+\alpha_{T} \rho_{0} c_{T}^{2} \delta T, \\
\delta e=\left(\frac{p_{0}}{\rho_{0}^{2}}-\frac{c_{p}-c_{V}}{\rho_{0} \alpha_{T}}\right) \delta \rho+c_{V} \delta T,
\end{gathered}
$$

and exploited the fact that MPCD fluids satisfy an ideal equation of state [30], which cancels the first term on the right-hand side of Eq. (16).

As a first approximation, we will study fluctuations occurring in an infinite system. In order to calculate the spectra of different hydrodynamic fluctuations, it will be convenient to introduce space and time-space Fourier transforms. The space Fourier transform of a field $f(\vec{r}, t)$ will be denoted by $\hat{f}=\hat{f}(\vec{k}, t)$, and the space-time Fourier transform of $f$ will be denoted by $\tilde{f}=\tilde{f}(\vec{k}, \omega)$. By Fourier transforming the fluctuating expressions Eqs. (11)-(13) we obtain the following equivalent relation:

$$
\mathbf{M}\left(\begin{array}{c}
\delta \tilde{\rho} \\
\delta \tilde{u}_{\|} \\
\delta \tilde{T}
\end{array}\right)=\left(\begin{array}{ccc}
-i \omega & \rho_{0} k & 0 \\
-\frac{c_{T}^{2} k}{\rho_{0}} & -i \omega+D_{l} k^{2} & -\alpha_{T} c_{T}^{2} k \\
0 & \frac{\gamma-1}{\alpha_{T}} k & -i \omega+\gamma D_{T} k^{2}+\frac{1}{\tau}
\end{array}\right)\left(\begin{array}{c}
\delta \tilde{\rho} \\
\delta \tilde{u}_{\|} \\
\delta \tilde{T}
\end{array}\right)=-\frac{1}{\rho_{0}}\left(\begin{array}{c}
0 \\
\frac{k_{i} k_{j}}{k} \tilde{\Sigma}_{i j} \\
\frac{i k_{i}}{c_{V}} \tilde{Q}_{i}-\frac{1}{c_{V} \tau} \delta \tilde{\mathcal{E}}
\end{array}\right)
$$

which defines the hydrodynamic matrix $\mathbf{M}=\mathbf{M}(\vec{k}, \omega)$, and

$$
\left(-i \omega+v k^{2}\right) \delta \tilde{u}_{\perp}=\frac{1}{\rho_{0} k_{\perp}}\left(k_{y} k_{j} \tilde{\Sigma}_{x j}-k_{x} k_{j} \tilde{\Sigma}_{y j}\right) .
$$

Here we have introduced the so called longitudinal kinematic viscosity, $D_{l}=(4 \eta+3 \zeta) / 3 \rho_{0}$, and the kinematic viscosity coefficient, $v=\eta / \rho_{0}$. We have also introduced a representation in terms of longitudinal, $\delta \tilde{u}_{\|}=i k_{j} \delta \tilde{u}_{j} / k$, and perpendicular, $\delta \tilde{u}_{\perp}=\left(k_{y} \delta \tilde{u}_{x}-k_{x} \delta \tilde{u}_{y}\right) / k_{\perp}$, fluctuating velocities, where $k_{\perp}=\left(k_{x}^{2}+k_{y}^{2}\right)^{1 / 2}$. We notice that since the fluid is isotropic, a second independent transverse velocity component exists that obeys an equation equivalent to Eq. (18).

The previous stochastic linearized equations reduce to the usual FH description in the limit of no application of the thermostat, i.e., for $\tau \rightarrow \infty$. A remarkable effect induced by the thermostat consists in modifying the relaxation rate of temperature fluctuations from its adiabatic value, $\gamma D_{T} k^{2}$, to the effective value, $\gamma D_{T} k^{2}+\tau^{-1}$. We will exploit this property subsequently, when we calculate the hydrodynamic modes of the thermostatted fluid.

In order to complete our model, the statistical properties of the noise terms $\Sigma_{i j}, Q_{i}$, and $\delta \mathcal{E}$ must be specified. As usual, $\Sigma_{i j}$ and $Q_{i}$ will be assumed to describe independent Gaussian Markovian processes with zero average [29,37,44]. Since it has been noted that the thermostat modifies the rate at which temperature fluctuations dissipate by introducing the additive term $\tau^{-1}$, we will propose that $Q_{i}$ satisfies the following Fluctuation-Dissipation Relation (FDR) in Fourier space,

$$
\begin{aligned}
k_{i} k_{j}^{\prime}\left\langle\tilde{Q}_{i}^{*}(\vec{k}, \omega) \tilde{Q}_{j}\left(\vec{k}^{\prime}, \omega^{\prime}\right)\right\rangle \\
\quad=2(2 \pi)^{4} k_{B} T_{0}^{2} \rho_{0} c_{V}\left(\gamma D_{T} k_{i} k_{i}^{\prime}+\frac{1}{\tau}\right) \delta\left(\omega-\omega^{\prime}\right) \delta\left(\vec{k}-\vec{k}^{\prime}\right),
\end{aligned}
$$

which reduces to the usual FDR for $Q_{i}$ in the limit of no application of the thermostat $[37,44]$. In the case of the stochastic stress tensor $\Sigma_{i j}$, we will assume the well-known form of the FDR $[29,37,44]$,

$$
\begin{aligned}
& \left\langle\tilde{\Sigma}_{i j}^{*}\left(\vec{k}^{\prime}, \omega^{\prime}\right) \tilde{\Sigma}_{k l}(\vec{k}, \omega)\right\rangle \\
& \quad=2(2 \pi)^{4} k_{B} T_{0} v_{i j k l} \delta\left(\omega-\omega^{\prime}\right) \delta\left(\vec{k}-\vec{k}^{\prime}\right) .
\end{aligned}
$$

Finally, the statistical properties of $\delta \mathcal{E}$ must be determined from the character of the applied thermostat, i.e., from the probability distribution Eq. (7). The application of the MBST as it was described in the previous section implies that $\delta \mathcal{E}$ also describes a Markovian process with vanishing first moment. In addition, $\delta \mathcal{E}$ is independent of both $Q_{i}$ and $\Sigma_{i j}$. As a first approximation, we will complete the statistical description of $\delta \mathcal{E}$ by assuming that it follows a Gaussian process, whose 
second moment will be written in the form

$$
\left\langle\delta \tilde{\mathcal{E}}^{*}\left(\vec{k}^{\prime}, \omega^{\prime}\right) \delta \tilde{\mathcal{E}}(\vec{k}, \omega)\right\rangle=(2 \pi)^{4} \sigma_{\text {therm }}^{2} \delta\left(\omega-\omega^{\prime}\right) \delta\left(\vec{k}-\vec{k}^{\prime}\right),
$$

where $\sigma_{\text {therm }}$ is a measure of the strength of the energy flow induced by the thermostat at the local level. The explicit form of $\sigma_{\text {therm }}$ is calculated in Appendix B.

The formalism presented above will be used in subsequent sections in order to model the behavior of spontaneous fluctuations in a thermostatted MPCD fluid. We will use it to calculate diverse dynamic correlation functions of the hydrodynamic fields, which are to be compared later with those observed in actual simulations.

\section{B. Strongly thermostatted case}

We will study first the effects of a STI, in which temperature fluctuations are forced to vanish as soon as they are produced. We will show that the application of the thermostat modifies the timescale over which temperature fluctuations evolve. This can be expected since in this case we may think of temperature fluctuations as if they decay very fast to equilibrium, say, on a timescale of order $\tau_{f}$, while the remaining hydrodynamic fluctuations vanish on a slower timescale, with a relaxation time $\tau_{s} \gg \tau_{f}$.

In order to illustrate that this argument is valid in MPCD simulations, let us consider a typical implementation of this method with the following input parameters: number of particles per cell $M=10$, mass of each particle $m=1$, collision angle $\alpha=130^{\circ}$, time step $\Delta t=0.1$, temperature $T=1$, cell size $a=1$, and $20^{3}=L^{3}$ collision cells. Notice that throughout this paper simulation units rather than physical units will be considered. Explicit expressions for the transport coefficients of MPCD fluids in terms of the previous parameters exist in diverse references [18,30-32], from which it can be anticipated that the shear viscosity of the simulated fluid (kinetic plus collisional contributions) will be $v \simeq 8.7 \times 10^{-1}$. Then velocity fluctuations with wavenumber $k=2 \pi / L$ will decay with a characteristic time $\tau_{\text {vel }}=1 /\left(v k^{2}\right) \simeq 1.1 \times 10^{1}$. In the absence of a thermostat, it can be also anticipated that the thermal diffusivity of the MPCD fluid will be $D_{T} \simeq 1.5 \times 10^{-1}$, and accordingly, the relaxation time for temperature fluctuations will be $\tau_{\text {temp }}=1 /\left(D_{T} k^{2}\right) \simeq 6.5 \times$ $10^{1}$. Both $\tau_{\text {vel }}$ and $\tau_{\text {temp }}$ are comparable. However, in the case of a STI, velocity fluctuations will still evolve slowly toward equilibrium while temperature fluctuations will be forced to decay much faster, in fact, in a time interval of size $\Delta t \ll \tau_{\mathrm{vel}}$.

Then the thermostat introduces a separation on the timescales for the relaxation of temperature with respect to the remaining hydrodynamic fields. This might be more clearly illustrated by writing Eq. (17) in terms of the effective relaxation rate for temperature fluctuations, $\tau_{\mathrm{eff}}^{-1}=\gamma D_{T} k^{2}+$ $\tau^{-1}$, and the variables with equal dimensions $\delta \bar{\rho}=\rho_{0}^{-1} \delta \hat{\rho}$, $\delta \bar{u}_{\|}=c_{T}^{-1} \delta \hat{u}_{\|}$, and $\delta \bar{T}=\alpha_{T} \delta \hat{T}$,

$$
\partial_{t}\left(\begin{array}{l}
\delta \bar{\rho} \\
\delta \bar{u}_{\|} \\
\delta \bar{T}
\end{array}\right)=-\left(\begin{array}{lcc}
0 & c_{T} k & 0 \\
-c_{T} k & D_{l} k^{2} & -c_{T} k \\
0 & (\gamma-1) c_{T} k & \tau_{\mathrm{eff}}^{-1}
\end{array}\right)\left(\begin{array}{l}
\delta \bar{\rho} \\
\delta \bar{u}_{\|} \\
\delta \bar{T}
\end{array}\right),
$$

where the elements of the matrix on the right-hand side have units of frequency and represent the inverse of the characteristic times involved in the evolution of the fluctuating fields. For simplicity, we have omitted in Eq. (22) the contribution of the stochastic forces.

Since $\tau_{\text {eff }}^{-1}$ is expected to be large, we can consider the limit $\tau_{\text {eff }}^{-1} \gg c_{T} k, D_{l} k^{2}$. It then follows that if a unit time of the order of $\tau_{\text {eff }}$, is used, the elements of the hydrodynamic matrix coupling $\delta \bar{\rho}$ and $\delta \bar{u}_{\|}$, with $\delta \bar{T}$, will be of the order of a small quantity $\varepsilon=c_{T} k \tau_{\text {eff }}$. This implies that for short timescales the evolution of $\delta \bar{\rho}$ and $\delta \bar{u}_{\|}$does not affect the motion of $\delta \bar{T}$. On the other hand, on a large timescale of the order of $1 / c_{T} k$, we expect that temperature fluctuations will have already decayed, and they will not perturb the motion of the slow variables $[45,46]$.

Indeed, the existence of well-defined timescales can be exploited to decouple the dynamics of slow and fast variables. For linear systems like the one described by Eq. (22), a systematic decoupling procedure of slow and fast variables has been developed by Geigenmüller, Titulaer, and Felderhof $[45,46]$. The application of this procedure to our case is presented in Appendix C. It yields the following reduced expression for the time evolution the slow variables $\delta \bar{\rho}$ and $\delta \bar{u}_{\|}$:

$$
\partial_{t}\left(\begin{array}{l}
\delta \bar{\rho} \\
\delta \bar{u}_{\|}
\end{array}\right)=-\left(1+\mathcal{O}\left(\varepsilon^{2}\right)\right)\left(\begin{array}{ll}
0 & c_{T} k \\
-c_{T} k & D_{l} k^{2}
\end{array}\right)\left(\begin{array}{l}
\delta \bar{\rho} \\
\delta \bar{u}_{\|}
\end{array}\right) .
$$

In a first approximation, we will neglect contributions of order $\varepsilon^{2}$ or higher. In this case, the linearized expression for density and longitudinal velocity fluctuations can be written in terms of a reduced hydrodynamic matrix $\mathbf{M}_{\text {red }}$ as

$$
\begin{aligned}
& \left(\begin{array}{cc}
-i \omega & \rho_{0} k \\
-\frac{c_{T}^{2} k}{\rho_{0}} & -i \omega+D_{l} k^{2}
\end{array}\right)\left(\begin{array}{c}
\delta \tilde{\rho} \\
\delta \tilde{u}_{\|}
\end{array}\right)=\mathbf{M}_{\mathrm{red}}\left(\begin{array}{c}
\delta \tilde{\rho} \\
\delta \tilde{u}_{\|}
\end{array}\right) \\
& =-\frac{1}{\rho_{0} k}\left(\begin{array}{c}
0 \\
k_{i} k_{j} \tilde{\Sigma}_{i j}
\end{array}\right),
\end{aligned}
$$

where we have reintroduced space-time Fourier transforms and the contributions of the stochastic currents.

The formal solutions of Eqs. (18) and (24) read

$$
\begin{gathered}
\delta \tilde{\rho}(\vec{k}, \omega)=-\frac{1}{\operatorname{det} \mathbf{M}_{\mathrm{red}}(\vec{k}, \omega)} k_{i} k_{j} \tilde{\Sigma}_{i j}(\vec{k}, \omega), \\
\delta \tilde{u}_{\|}(\vec{k}, \omega)=-\frac{i \omega}{\rho_{0} k \operatorname{det} \mathbf{M}_{\mathrm{red}}(\vec{k}, \omega)} k_{i} k_{j} \tilde{\Sigma}_{i j}(\vec{k}, \omega), \\
\delta \tilde{u}_{\perp}(\vec{k}, \omega)=-\frac{1}{\rho_{0} k_{\perp}\left(-i \omega+v k^{2}\right)}\left[k_{y} k_{j} \tilde{\Sigma_{x j}}(\vec{k}, \omega)\right. \\
\left.-k_{x} k_{j} \tilde{\Sigma}_{y j}(\vec{k}, \omega)\right] .
\end{gathered}
$$

Equation (27) shows that transverse velocity fluctuations are not affected by the application of the thermostat. In Eqs. (25) and (26), det $\mathbf{M}_{\text {red }}$ is a function of $\vec{k}$ and $\omega$, which can be written in the form

$$
\operatorname{det} \mathbf{M}_{\mathrm{red}}(\vec{k}, \omega)=\left[-i \omega-s_{2, \mathrm{red}}(\vec{k})\right]\left[-i \omega-s_{3, \mathrm{red}}(\vec{k})\right],
$$


where $s_{2 \text {,red }}(\vec{k})$ and $s_{3, \text { red }}(\vec{k})$ are the longitudinal hydrodynamic modes of the strongly thermostatted system. They have the explicit form

$$
\begin{aligned}
& s_{2, \text { red }}(\vec{k})=i c_{T} k\left[1-\left(\frac{D_{l} k}{2 c_{T}}\right)^{2}\right]^{1 / 2}-\frac{1}{2} D_{l} k^{2}, \\
& s_{3, \text { red }}(\vec{k})=-i c_{T} k\left[1-\left(\frac{D_{l} k}{2 c_{T}}\right)^{2}\right]^{1 / 2}-\frac{1}{2} D_{l} k^{2} .
\end{aligned}
$$

Equations (25)-(30) together with the FDR, Eq. (20), can be used to calculate the dynamic correlation functions of $\delta \tilde{\rho}$, $\delta \tilde{u}_{\|}$, and $\delta \tilde{u}_{\perp}$. In this work we will be interested in calculating only correlations of longitudinal variables since $\delta \tilde{u}_{\perp}$ is not affected by the thermostat. Let us consider first the density autocorrelation function or dynamic structure factor,

$$
S_{\rho \rho}(\vec{k}, \omega)=\left\langle\delta \hat{\rho}^{*}(\vec{k}, 0) \delta \tilde{\rho}(\vec{k}, \omega)\right\rangle,
$$

which is the most relevant quantity in light scattering experiments in liquids and liquid mixtures [38,39]. From Eqs. (20) and (25) we obtain

$$
S_{\rho \rho}(\vec{k}, \omega)=2(2 \pi)^{3} \delta_{\vec{k}}(0) k_{B} T_{0} \frac{\rho_{0} D_{l} k^{2}}{4 c_{T}^{2}}\left[\frac{1+\omega_{s, \text { red }}^{-1}\left(\omega+\omega_{s, \text { red }}\right)}{\left(\omega+\omega_{s, \text { red }}\right)^{2}+\Gamma_{\text {red }}^{2}}+\frac{1-\omega_{s, \text { red }}^{-1}\left(\omega-\omega_{s, \text { red }}\right)}{\left(\omega-\omega_{s, \text { red }}\right)^{2}+\Gamma_{\text {red }}^{2}}\right],
$$

where we have introduced the following notation:

$$
\begin{gathered}
\omega_{s, \text { red }}=\omega_{s, \text { red }}(k)=\operatorname{Im} s_{2, \text { red }}(k)=c_{T} k\left[1-\left(\frac{D_{l} k}{2 c_{T}}\right)^{2}\right]^{1 / 2}, \\
\Gamma_{\text {red }}=\Gamma_{\text {red }}(k)=-\operatorname{Re} s_{2, \text { red }}(k)=\frac{1}{2} D_{l} k^{2}
\end{gathered}
$$

Moreover, $\delta_{\vec{k}}(0)$ in Eq. (32) is a formal representation of the Dirac delta function in $\vec{k}$-space evaluated at $\vec{k}=0$. Notice that for large systems $\delta_{\vec{k}}(0) \simeq(2 \pi)^{-3} V$, where $V$ is the scattering volume.

Thus, the dynamic structure factor of a thermostatted fluid consists of two peaks located at $\pm \omega_{s, \text { red }}$ (Brillouin peaks). The usual central peak (thermal or Rayleigh peak) has been suppressed as a consequence of the application of the thermostat. In addition, notice that in the hydrodynamic limit $c_{T} k \gg D_{l} k^{2}$, we have $\omega_{s, \text { red }} \simeq c_{T} k$, and these peaks reduce to two Lorentzians, which result from modes propagating at the isothermal sound speed. In Eq. (32) this limit has not been taken into account and the Lorentzians are slightly asymmetric with respect to $\pm \omega_{s, \text { red }}$, but the dynamic structure factor is still symmetric as a whole.

In the following, it will be convenient to normalize the longitudinal correlation functions with respect to the area under $S_{\rho \rho}$ in $\omega$ space:

$$
I_{\rho \rho}(\vec{k})=\int d \omega S_{\rho \rho}(\vec{k}, \omega)
$$

The normalized density autocorrelation, $\bar{S}_{\rho \rho}=S_{\rho \rho} / I_{\rho \rho}$, reads

$$
\begin{aligned}
\bar{S}_{\rho \rho} & =\frac{D_{l} k^{2}}{4 \pi}\left\{\frac{1}{\left(\omega+\omega_{s, \text { red }}\right)^{2}+\Gamma_{\text {red }}^{2}}+\frac{1}{\left(\omega-\omega_{s, \text { red }}\right)^{2}+\Gamma_{\text {red }}^{2}}\right. \\
& \left.+\frac{1}{\omega_{s, \text { red }}}\left[\frac{\omega+\omega_{s, \text { red }}}{\left(\omega+\omega_{s, \text { red }}\right)^{2}+\Gamma_{\text {red }}^{2}}-\frac{\omega-\omega_{s, \text { red }}}{\left(\omega-\omega_{s, \text { red }}\right)^{2}+\Gamma_{s, \text { red }}^{2}}\right]\right\} .
\end{aligned}
$$

A completely analogous calculation leads to the normalized longitudinal velocity autocorrelation function,

$$
\bar{S}_{u_{\|} u_{\|}}(\vec{k}, \omega)=\frac{\left\langle\delta \hat{u}_{\|}^{*}(\vec{k}, 0) \delta \tilde{u}_{\|}(\vec{k}, \omega)\right\rangle}{I_{\rho \rho}(\vec{k})}=\frac{\omega^{2}}{\rho_{0}^{2} k^{2}} \bar{S}_{\rho \rho}(\vec{k}, \omega),
$$

and to the density-velocity cross-correlation function,

$$
\bar{S}_{\rho u_{\|}}(\vec{k}, \omega)=\frac{\left\langle\delta \hat{\rho}^{*}(\vec{k}, 0) \delta \tilde{u}_{\|}(\vec{k}, \omega)\right\rangle}{I_{\rho \rho}(\vec{k})}=i \frac{\omega}{\rho_{0} k} \bar{S}_{\rho \rho}(\vec{k}, \omega),
$$

with $\left\langle\delta \hat{u}_{\|}^{*}(\vec{k}, 0) \delta \tilde{\rho}(\vec{k}, \omega)\right\rangle=-\left\langle\delta \hat{\rho}^{*}(\vec{k}, 0) \delta \tilde{u}_{\|}(\vec{k}, \omega)\right\rangle$.

Although $\bar{S}_{u_{\|} u_{\|}}$and $\bar{S}_{\rho u_{\|}}$cannot be directly explored in experiments, they can be easily measured in MPCD simulations. Measurements of these functions will be presented in subsequent sections.

\section{Partially thermostatted case}

We shall consider now spontaneous fluctuations in a fluid where a thermostat is applied at regular time intervals. The analysis of the previous section suggested that in strongly thermostatted systems the effective relaxation rate of the temperature fluctuations in the fluid can take significant small values in such a way that the timescale for the relaxation of temperature fluctuations is very short when compared with the remaining characteristic times in the system. Let us consider in detail the opposite case, i.e., when no thermostat is applied at all. In MPCD $D_{T}, D_{l}$, and $c_{T}$ obey the usual relation of the hydrodynamic limit $D_{T} k^{2}, D_{l} k^{2} \ll c_{T} k$. This can be illustrated for a typical MPCD simulation performed with the same parameters discussed in the previous section for which $D_{T} k^{2} \sim$ $1.5 \times 10^{-2}$ and $D_{l} k^{2} \sim v k^{2} \sim 9 \times 10^{-2}$. Since a MPCD fluid has an ideal equation of state [30], $c_{T}=\sqrt{k_{B} T / m}$, then $c_{T} k \sim 3.1 \times 10^{-1}$, from which the hydrodynamic limit is shown to be a good approximation. Thus, it can be intuitively expected that the application of the thermostat in regular time intervals of size $\tau=n_{\text {therm }} \Delta t$ will modify the temperature relaxation rate from its nonthermostatted value $D_{T} k^{2} \ll c_{T} k$, for $n_{\text {therm }} \rightarrow \infty$, to a maximum value $D_{T} k^{2}+\tau^{-1} \gg c_{T} k$, for $n_{\text {therm }}=1$. The latter case was analyzed in detail in the previous section. Accordingly, in order to describe thermal 
fluctuations in partially thermostatted systems it is necessary to extend the linearized theory to consider thermal fluctuations whose relaxation times are not restricted by the hydrodynamic limit but may take arbitrary values. Here we shall consider this extension.

We notice first that the general solution for $\delta \tilde{\rho}$ in terms of the stochastic heat and momentum currents is [Eq. (17)]

$$
\begin{aligned}
\delta \tilde{\rho}= & \frac{1}{\operatorname{det} \mathbf{M}}\left[-\left(-i \omega+\gamma D_{T} k^{2}+\tau^{-1}\right) k_{i} k_{j} \tilde{\Sigma}_{i j}\right. \\
& \left.+\frac{\alpha_{T} c_{T}^{2} k^{2}}{c_{V}}\left(i k_{i} \tilde{Q}_{i}-\tau^{-1} \delta \tilde{\mathcal{E}}\right)\right] .
\end{aligned}
$$

Using the FDR in Fourier space, Eqs. (19)-(21), the density autocorrelation function is found to be

$$
\begin{aligned}
S_{\rho \rho}(\vec{k}, \omega)= & \frac{2(2 \pi)^{4} k_{B} T_{0} \delta_{\vec{k}}(0)}{|\operatorname{det} \mathbf{M}(\vec{k}, \omega)|^{2}} \rho_{0} k^{4} D_{l}\left[\omega^{2}+\left(\gamma D_{T} k^{2}+\tau^{-1}\right)^{2}\right. \\
& +\frac{(\gamma-1) c_{T}^{2}}{D_{l}}\left(\gamma D_{T} k^{2}+\tau^{-1}\right) \\
& \left.+\frac{(\gamma-1) c_{T}^{2}}{2 D_{l} \rho_{0} c_{V}} \frac{\sigma_{\text {therm }}^{2}}{k_{B} T_{0}^{2} \tau^{2}}\right]
\end{aligned}
$$

In these expressions the quantity $\operatorname{det} \mathbf{M}(\vec{k}, \omega)$ can be written in the form

$$
\operatorname{det} \mathbf{M}(\vec{k}, \omega)=\left[-i \omega-s_{1}(\vec{k})\right]\left[-i \omega-s_{2}(\vec{k})\right]\left[-i \omega-s_{3}(\vec{k})\right],
$$

where the functions of the wavevector $s_{1}, s_{2}$, and $s_{3}$, represent the hydrodynamic modes of the partially thermostatted fluid. For simplicity, we will find their explicit expressions by following a resembling procedure to the one used in linearized hydrodynamics. That is, we will restrict ourselves to the case in which sound propagation is a much faster process than momentum and heat diffusion, i.e., $c_{T} k \gg D_{l} k^{2}, D_{T} k^{2}$. Then we propose that $s_{1}, s_{2}$, and $s_{3}$, can be written as an expansion $s_{i}=s_{i}^{(0)}+s_{i}^{(1)}+\cdots$, for $i=1,2,3$, where $s_{i}^{(n)}$ is a term of order $n$ in the small frequencies $D_{l} k^{2}$ and $D_{T} k^{2}$. In Appendix D we present in detail the calculation of the hydrodynamic modes of the thermostatted fluid. The contributions of order zero in $D_{l} k^{2}$ and $D_{T} k^{2}$ read

$$
\begin{gathered}
s_{1}^{(0)}=B-\frac{1}{3} \tau^{-1}, \\
s_{2}^{(0)}=-\frac{1}{2}(B-\sqrt{3} i A)-\frac{1}{3} \tau^{-1}, \\
s_{3}^{(0)}=-\frac{1}{2}(B+\sqrt{3} i A)-\frac{1}{3} \tau^{-1},
\end{gathered}
$$

where the quantities $A$ and $B$ are functions of $k, c_{T}$ and $D_{T}$ given by Eqs. (D3)-(D6).

The contributions of first order in $D_{l} k^{2}$ and $D_{T} k^{2}$ can be written in the form

$$
s_{i}^{(1)}=-k^{2} \frac{\gamma D_{T}\left(s_{i}^{(0) 2}+c_{T}^{2} k^{2}\right)+D_{l}\left(s_{i}^{(0) 2}+s_{i}^{(0)} \tau^{-1}\right)}{3 s_{i}^{(0) 2}+2 s_{i}^{(0)} \tau^{-1}+\gamma c_{T}^{2} k^{2}},
$$

for $i=1,2,3$. It can be shown that if $\tau \rightarrow \infty$, the usual hydrodynamic modes can be recovered from Eqs. (42)-(45); i.e., for $c_{T} k \tau \gg 1$, we have $s_{1} \simeq-D_{T} k^{2}$ and $s_{2,3} \simeq \mp i c_{s} k-$ $\Gamma k^{2}$, where $c_{s}=\gamma^{1 / 2} c_{T}$ is the isentropic sound speed and $\Gamma=\left[D_{l}+(\gamma-1) D_{T}\right] / 2$ is the sound attenuation coefficient.
Then, Eqs. (42)-(45) contain the hydrodynamic limit as special case and can be used also when $\tau^{-1}$ takes large values, which is the case of a strongly thermostatted system.

We also notice that for the values of the material parameters of an MPCD fluid, $s_{1}$, Eqs. (42) and (45), has no imaginary part. This can be anticipated from the symmetry of the problem in which two propagating and one diffusive isotropic modes are expected. This allow us to write $\operatorname{det} \mathbf{M}(\vec{k}, \omega)$ in the form

$$
\begin{aligned}
\operatorname{det} & \mathbf{M}(\vec{k}, \omega) \\
= & \left(-i \omega-\omega_{T}\right)\left[-i\left(\omega-\omega_{s}\right)-\omega_{\Gamma}\right]\left[-i\left(\omega+\omega_{s}\right)-\omega_{\Gamma}\right],
\end{aligned}
$$

where $\quad \omega_{T}=\omega_{T}(\vec{k})=\operatorname{Re}\left(s_{1}\right), \quad \omega_{s}=\omega_{s}(\vec{k})=\operatorname{Im}\left(s_{3}\right)=$ $-\operatorname{Im}\left(s_{2}\right)$, and $\omega_{\Gamma}=\omega_{\Gamma}(\vec{k})=\operatorname{Re}\left(s_{2}\right)=\operatorname{Re}\left(s_{3}\right)$.

The final expression for the density-density correlation function can be found by substituting Eq. (46) into Eq. (40) and performing a partial fraction decomposition of the result. This procedure yields

$$
\begin{aligned}
S_{\rho \rho}(\vec{k}, \omega)= & 2(2 \pi)^{3} \delta_{\vec{k}}(0) k_{B} T_{0} \rho_{0} D_{l} k^{4}\left\{\frac{\bar{\alpha}}{\omega^{2}+\omega_{T}^{2}}\right. \\
& +\bar{\beta}\left[\frac{1}{\left(\omega+\omega_{s}\right)^{2}+\omega_{\Gamma}^{2}}+\frac{1}{\left(\omega-\omega_{s}\right)^{2}+\omega_{\Gamma}^{2}}\right] \\
& \left.+\bar{\gamma}\left[\frac{\omega+\omega_{s}}{\left(\omega+\omega_{s}\right)^{2}+\omega_{\Gamma}^{2}}-\frac{\omega-\omega_{s}}{\left(\omega-\omega_{s}\right)^{2}+\omega_{\Gamma}^{2}}\right]\right\},
\end{aligned}
$$

where the following notation has been introduced:

$$
\begin{gathered}
\bar{\beta}=\frac{1}{2 K}\left[\omega_{T}^{2}+\omega_{s}^{2}+\omega_{\Gamma}^{2}-\frac{\bar{\lambda}}{\omega_{s}^{2}+\omega_{\Gamma}^{2}}\left(-\omega_{s}^{2}+3 \omega_{\Gamma}^{2}-\omega_{T}^{2}\right)\right] \\
\bar{\gamma}=\frac{1}{2 K \omega_{s}}\left[-\omega_{T}^{2}+\omega_{s}^{2}+\omega_{\Gamma}^{2}\right. \\
\left.+\frac{\bar{\lambda}}{\omega_{s}^{2}+\omega_{\Gamma}^{2}}\left(3 \omega_{s}^{2}-\omega_{\Gamma}^{2}+\omega_{T}^{2}\right)\right] \\
\bar{\alpha}=-2 \bar{\beta}+2 \omega_{s} \bar{\gamma},
\end{gathered}
$$

and

$$
\begin{gathered}
K=\left(3 \omega_{s}^{2}-\omega_{\Gamma}^{2}+\omega_{T}^{2}\right)\left(\omega_{T}^{2}+\omega_{s}^{2}+\omega_{\Gamma}^{2}\right) \\
-\left(-\omega_{s}^{2}+3 \omega_{\Gamma}^{2}-\omega_{T}^{2}\right)\left(\omega_{T}^{2}-\omega_{s}^{2}-\omega_{\Gamma}^{2}\right), \\
\bar{\lambda}=\gamma^{2} D_{T}^{2} k^{4}+\gamma(\gamma-1) c_{T}^{2} k^{2} \frac{D_{T}}{D_{l}}+\frac{(\gamma-1) c_{T}^{2}}{2 D_{l} \rho_{0} c_{V}} \frac{\sigma_{\text {therm }}^{2}}{k_{B} T_{0}^{2} \tau^{2}} .
\end{gathered}
$$

Equations (42)-(52) define the dynamic structure factor of a partially thermostatted system. Details apart, Eq. (47) has the same structure as the dynamic structure factor of a liquid in thermal equilibrium [38]. It consists of three peaks located at $\omega=0$ and $\omega= \pm \omega_{s}$. The factor proportional to $\bar{\gamma}$ in Eq. (47) describes an asymmetry of the lateral peaks with respect to their maxima; however, the spectrum of density fluctuations is symmetric as a whole.

The location of the Brillouin peaks, their maxima, and the maximum of the Rayleigh peak change as a function of $\tau$, through the dependence of $\omega_{T}, \omega_{s}$, and $\omega_{\Gamma}$ on this quantity. Two important limiting cases can be obtained from 
Eqs. (42)-(52). We notice first that in the adiabatic case, in which holds the limit $\tau c_{T} k \gg 1$, we have $\omega_{l} \simeq-D_{T} k^{2}, \omega_{s} \simeq$ $c_{s} k, \omega_{\Gamma} \simeq-\Gamma k^{2}$, and consequently $\bar{\alpha} \simeq(\gamma-1) D_{T} / c_{s}^{2} k^{2} D_{l}$, $\bar{\beta} \simeq \Gamma / 2 c_{s}^{2} D_{l} k^{2}$, and $\bar{\gamma} \simeq\left(D_{l}+3(\gamma-1) D_{T}\right) / 4 c_{s}^{3} k^{3}$. Using these results, the well-known expression for the dynamic structure factor [38] is recovered:

$$
\begin{aligned}
S_{\rho \rho}(\vec{k}, \omega)= & \frac{2(2 \pi)^{3} k_{B} T_{0} \rho_{0} \delta_{\vec{k}}(0)}{c_{s}^{2}}\left\{\frac{(\gamma-1) D_{T} k^{2}}{\omega^{2}+D_{T}^{2} k^{4}}+\frac{1}{2} \Gamma k^{2}\left[\frac{1}{\left(\omega+c_{s} k\right)^{2}+\Gamma^{2} k^{4}}+\frac{1}{\left(\omega-c_{s} k\right)^{2}+\Gamma^{2} k^{4}}\right]\right. \\
& \left.+\frac{3 \Gamma-D_{l}}{2} k^{2}\left[\frac{\omega+c_{s} k}{\left(\omega+c_{s} k\right)^{2}+\Gamma^{2} k^{4}}-\frac{\omega-c_{s} k}{\left(\omega-c_{s} k\right)^{2}+\Gamma^{2} k^{4}}\right]\right\} .
\end{aligned}
$$

On the other hand, for a strongly thermostatted system for which it is expected that $\tau c_{T} k \ll 1$, we obtain (up the smallest order in the quantity $\left.c_{T} k / D_{T} k^{2}\right), \omega_{T} \simeq-\gamma D_{T} k^{2}, \omega_{\Gamma} \simeq$ $-D_{l} k^{2} / 2=-\Gamma_{\text {red }}, \omega_{s} \simeq-c_{T} k \simeq-\omega_{\text {(red) }}$, and $\bar{\beta} \simeq 1 / 4 c_{T}^{2} k^{2}$, $\bar{\gamma} \simeq 1 / 4 c_{T}^{3} k^{3}, \bar{\alpha} \simeq 0$. Using these results we recover the expression for the dynamic structure factor in the STI, Eqs. (32)-(34), with the only difference being the absence of terms order $D_{l} k^{2} / c_{T} k$. This difference can be expected because the thermostatted modes $s_{1}, s_{2}$, and $s_{3}$, were calculated under the assumption $D_{l} k^{2} / c_{T} k \ll 1$.

In general, when the time interval for the application of the thermostat $\tau$ changes from $\tau=\Delta t$ to $\tau \rightarrow \infty$, the central peak of $S_{\rho \rho}$ shrinks, while the lateral peaks change their location from $\omega \simeq c_{s} k$ to $\omega \simeq c_{T} k$, and consequently they get closer. These effects will be explicitly shown in subsequent sections. Finally, in order to compare our results with numerical simulations, it will be convenient to introduce the normalized dynamic structure factor $\bar{S}_{\rho \rho}=S_{\rho \rho} / I_{\rho \rho}$ :

$$
\begin{aligned}
\bar{S}_{\rho \rho}= & \frac{1}{\pi\left(\bar{\alpha}\left|\omega_{T}\right|^{-1}+2 \bar{\beta}\left|\omega_{\Gamma}\right|^{-1}\right)}\left\{\frac{\bar{\alpha}}{\omega^{2}+\omega_{T}^{2}}\right. \\
& +\bar{\beta}\left[\frac{1}{\left(\omega+\omega_{s}\right)^{2}+\omega_{\Gamma}^{2}}+\frac{1}{\left(\omega-\omega_{s}\right)^{2}+\omega_{\Gamma}^{2}}\right] \\
& \left.+\bar{\gamma}\left[\frac{\omega+\omega_{s}}{\left(\omega+\omega_{s}\right)^{2}+\omega_{\Gamma}^{2}}-\frac{\omega-\omega_{s}}{\left(\omega-\omega_{s}\right)^{2}+\omega_{\Gamma}^{2}}\right]\right\}
\end{aligned}
$$

For simplicity, we will restrict the analysis of dynamic correlations in PTI to the calculation of $S_{\rho \rho}$. The remaining correlation functions can be studied analogously.

\section{MEASUREMENTS}

\section{A. Strongly thermostatted case}

We performed simulations in order to calculate numerically the correlation functions of spontaneous fluctuations in a MPCD fluid. As basic units we fix the mass of the particles at $m=1$, the size of the collisions cells at $a=1$, and the time step at $\Delta t=0.1$. We choose the rotation angle, $\alpha$, the number density (average number of particles per cell) $M$, and the mean-free path, $\lambda=a \Delta t\left(k_{B} T_{0} / m\right)^{1 / 2}$, as the independent parameters characterizing each simulation. Simulations were performed for a fluid enclosed in a three-dimensional box with a total of $L^{3}=20^{3}$ collision cells for a fixed mean-free path $\lambda=1$. At a first stage, we choose a collision angle $\alpha=160^{\circ}$ and three different densities, $M=5,10,20$. The thermostating procedure described in Sec. II A, was performed with $T_{0}=1$. Two remarks concerning this selection for the values of the MPCD parameters are worth stressing. First, notice that for the selected densities, fluids with significant different viscosities will be simulated. In fact, if these fluids were simulated under flow conditions, the Reynold's numbers of those with $M=10$ and 20 would be approximately 2 and 5 times larger than that of the fluid with $M=5$, respectively. Second, the parameters have been selected in such a way that the simulated MPCD fluid will be in the so-called liquid regime, where collisional effects dominate over kinetic transport [28], as can be observed by inspection of Eqs. (54), (55), (57), and (58) below.

During simulations, after allowing for relaxation of the system, hydrodynamic fluctuating fields are measured at the cell level and at each time step. In this work, Fourier transforms of these fields are approximated by the corresponding discrete Fourier transforms. Time series containing $10^{6}$ successive space Fourier transforms of density and velocities are stored in order to calculate dynamic correlation functions. The main purpose of this section is to compare these correlations with those given theoretically by Eqs. (36)-(38). In order to perform this comparison quantitatively $\bar{S}_{\rho \rho}, \bar{S}_{\rho u_{\|}}$, and $\bar{S}_{u_{\|} u_{\|}}$must be evaluated, which in turn requires knowledge of the value of diverse material parameters and transport coefficients of the simulated fluid, e.g., $c_{V}, \gamma, c_{T}, D_{l}$, and $\nu$. These properties have been calculated for a fluid of Malevanets and Kapral in terms of $\Delta t, \alpha, a$, and $M$, by using different approaches [18,30-32]. It is well established that MPCD fluids have an ideal equation of state, i.e., $c_{V}=3 k_{B} / 2, \gamma=5 / 3, c_{T}=\sqrt{k_{B} T / m}$, and a kinematic viscosity coefficient that can be split into a kinetic and a collisional contributions $v=v^{\text {kin }}+v^{\text {col }}$, with [30]

$$
v^{v^{\text {kin }}}=\frac{k_{B} T_{0} \Delta t}{2 m}\left\{\frac{5 M}{\left(M-1+e^{-M}\right)[2-\cos (\alpha)-\cos (2 \alpha)]}-1\right\}
$$

and

$$
v^{\mathrm{col}}=\frac{a^{2}}{18 M \Delta t}\left(M-1+e^{-M}\right)[1-\cos (\alpha)] .
$$

It is worth mentioning also that since angular momentum is not preserved by the present MPCD algorithm [47], the bulk viscosity is zero, and the longitudinal kinematic viscosity has the following expression in terms of $v^{\mathrm{kin}}$ and $v^{\mathrm{col}}$ :

$$
D_{l}=\frac{4}{3} v^{\mathrm{kin}}+v^{\mathrm{col}} \text {. }
$$


We will use the previous expressions to evaluate the normalized dynamic correlations given by Eqs. (36)-(38) and perform the comparison against simulations. Figure 1 shows a comparison of the theoretical density autocorrelation function $\bar{S}_{\rho \rho}$, Eq. (36), with the results of the simulation. The spectra presented in Fig. 1 correspond to wavevectors with magnitude $k=2 \pi / L$. Different orientations for the wavevector were probed to verify that the isotropy of the system was preserved in our simulations. It can be observed that this correlation function exhibits the properties expected from Eq. (36). A very good agreement is found for the three considered densities $M=5,10,20$.

Figures 2 and 3 present a comparison of the remaining normalized longitudinal correlation functions $\bar{S}_{u_{\|} u_{\|}}$, Eq. (37), and $\operatorname{Im}\left\{\bar{S}_{\rho u_{\|}}\right\}$, Eq. (38), for simulations of a system with numerical density $M=10$ and collision angle $\alpha=160^{\circ}$. A very good agreement is also observed in these cases between simulations and expressions obtained from thermostatted FH.

\section{B. Partially thermostatted case}

In order to test the PTI situation, we also performed simulations for a system in which the thermostat is applied only every $n_{\text {therm }}=\tau / \Delta t$ time steps. The system parameters used for this set of simulations are $M=10, \alpha=130^{\circ}$, and $\Delta t=0.1$. We notice that in the PTI the value of the thermal diffusivity $D_{T}$ is required in order to compare the model yielding Eq. (53) with simulation results. In the absence of a thermostat the thermal diffusivity of the MPCD fluid is $D_{T}=D_{T}^{\mathrm{kin}}+D_{T}^{\mathrm{col}}$, where the kinetic and a collisional contributions are given in terms of $T_{0}, \Delta t, \alpha, M, a$, and $m$, by [30]

$$
\begin{gathered}
D_{T}^{\mathrm{kin}}=\frac{k_{B} T_{0} \Delta t}{2 m}\left\{\frac{3}{1-\cos \alpha}-1+\frac{6}{M}\left[\frac{4}{5}-\frac{1}{4} \csc ^{2}(\alpha / 2)\right]\right\} \\
D_{T}^{\mathrm{col}}=\frac{a^{2}}{15 M \Delta t}\left(1-\frac{1}{M}\right)[1-\cos (\alpha)]
\end{gathered}
$$

respectively.

The results of the previous section show that the application of the thermostat does not modify the longitudinal kinematic viscosity coefficient appreciably, even in the STI. This can be readily seen in Fig. 1, where the height and half width of the peaks can be very well fitted by using Eqs. (54)-(56), which have been obtained for constant energy simulations. Therefore, momentum transport between cells is only slightly modified by thermostatted dynamics. In order to simplify our following analysis, we shall assume that the same is true for energy transport. Consequently, we propose that the transport coefficients in thermostatted MPCD have the same values as they have in the adiabatic version of the method. This assumption is also justified by the good agreement found subsequently between our analysis and numerical results.

With the purpose of calculating the normalized dynamic structure factor of a partially thermostatted system, $\bar{S}_{\rho \rho}$ in Eq. (53), we replace $D_{T}=D_{T}^{\text {kin }}+D_{T}^{\text {col }}$, given by Eqs. (57)(58) and $D_{l}$, given by Eqs. (54)-(56), into the expressions for hydrodynamic modes, Eqs. (42)-(45). These results together with Eq. (B7) for $\sigma_{\text {therm }}^{2}$ are used to calculate the quantities
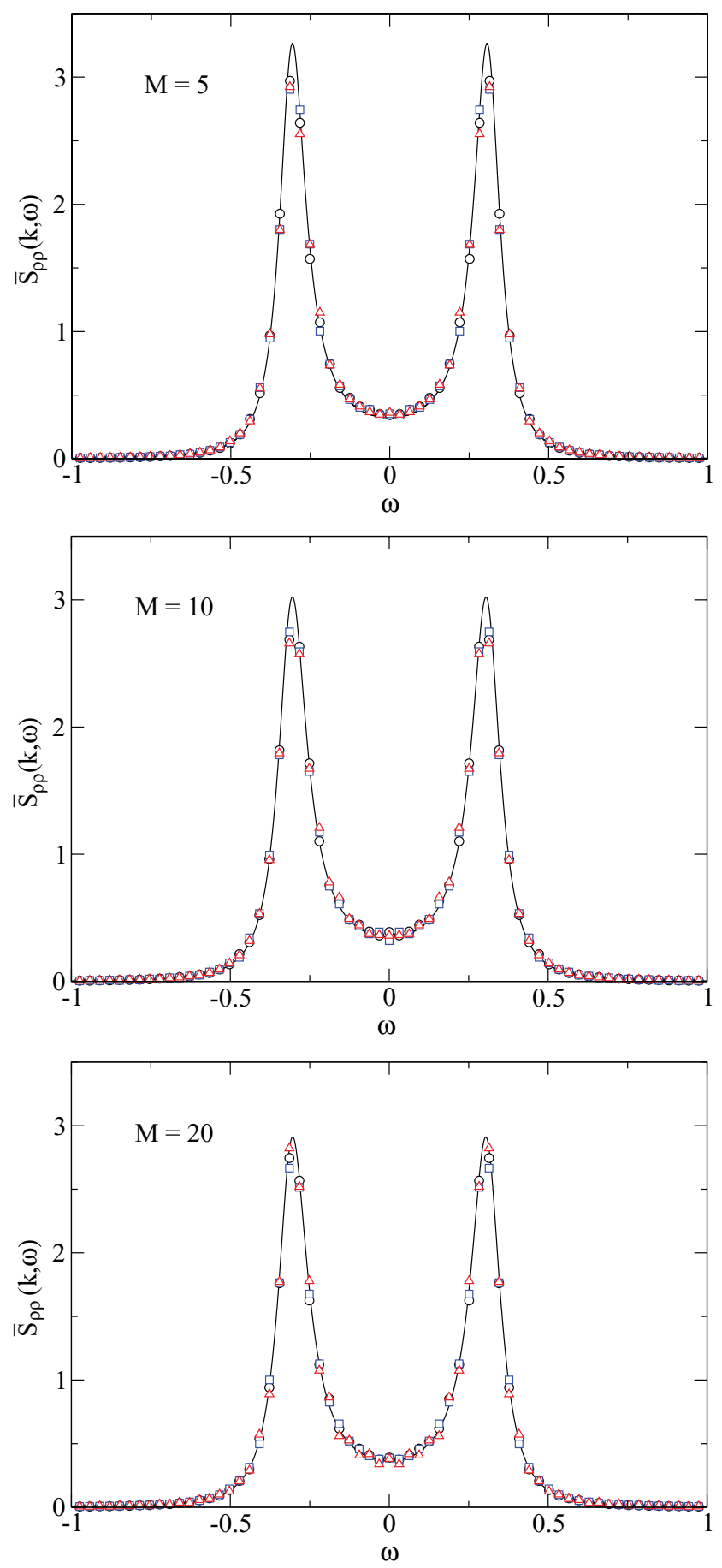

FIG. 1. (Color online) Dynamic structure factor of a MPCD fluid with three different numerical densities $M=5,10,20$. Continuous lines correspond to the density autocorrelation function obtained from thermostatted FH, Eq. (36). Symbols correspond to numerical simulations for wavevectors $\vec{k}=\frac{2 \pi}{L}(1,0,0)$ (circles), $\vec{k}=\frac{2 \pi}{L}(0,1,0)$ (squares), and $\vec{k}=\frac{2 \pi}{L}(0,0,1)$ (triangles).

defining the width and maxima of the thermal and sound peaks, Eqs. (48)-(52). $\bar{S}_{\rho \rho}$ is calculated both analytically and numerically for different values of $n_{\text {therm }}=1,10,100$, and 1000 , and for no thermostat at all.

The comparison between simulations and theory is shown in Fig. 4. We observe that our model reproduces very well 


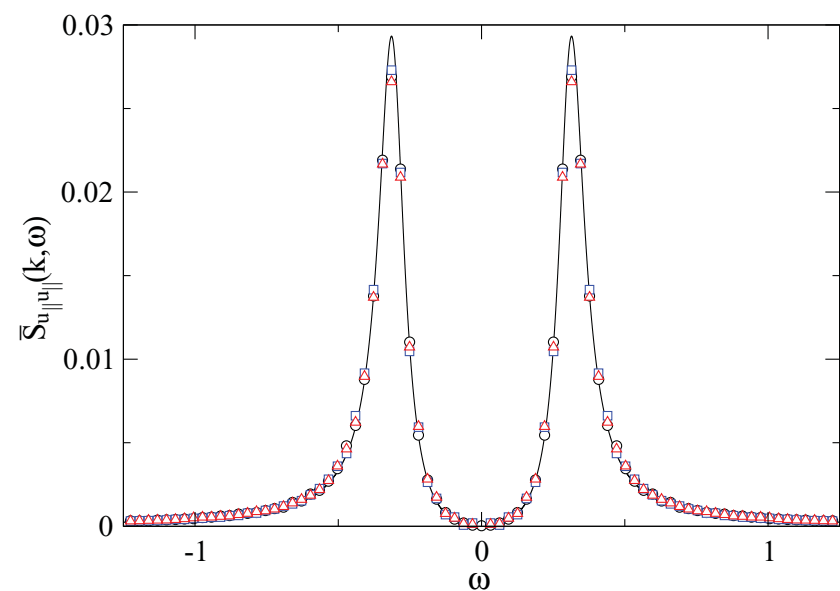

FIG. 2. (Color online) Longitudinal velocity autocorrelation function of a thermostatted MPCD fluid. Continuous line corresponds to the prediction of thermostatted FH, Eq. (37). Symbols correspond to numerical simulations of a fluid with numerical density $M=10$, collision angle $\alpha=160^{\circ}$, for wavevectors $\vec{k}=\frac{2 \pi}{L}(1,0,0)$ (circles), $\vec{k}=\frac{2 \pi}{L}(0,1,0)$ (squares), and $\vec{k}=\frac{2 \pi}{L}(0,0,1)$ (triangles).

the general behavior of the observed spectra. Figure 4 shows that when $n_{\text {therm }}$ changes from $n_{\text {therm }}=1$ to $n_{\text {therm }} \rightarrow \infty$, the lateral peaks shrink initially and eventually increase by a small amount in order to reach their final height. They also change their position from $\omega= \pm c_{T} k$ to $\omega= \pm c_{s} k$. On the other hand, the central peak is negligible initially and increases gradually up to its maximum value. All these features are reproduced satisfactorily by our approach. We also notice that there is a very good quantitative agreement between our model and the simulations in the cases of strong and weak thermostatting interactions.

It should be noticed that the hydrodynamic approximation $c_{T} k \gg D_{l} k^{2}$ is not longer valid in MPCD if perturbations propagating with wavenumbers relatively larger than $k=$

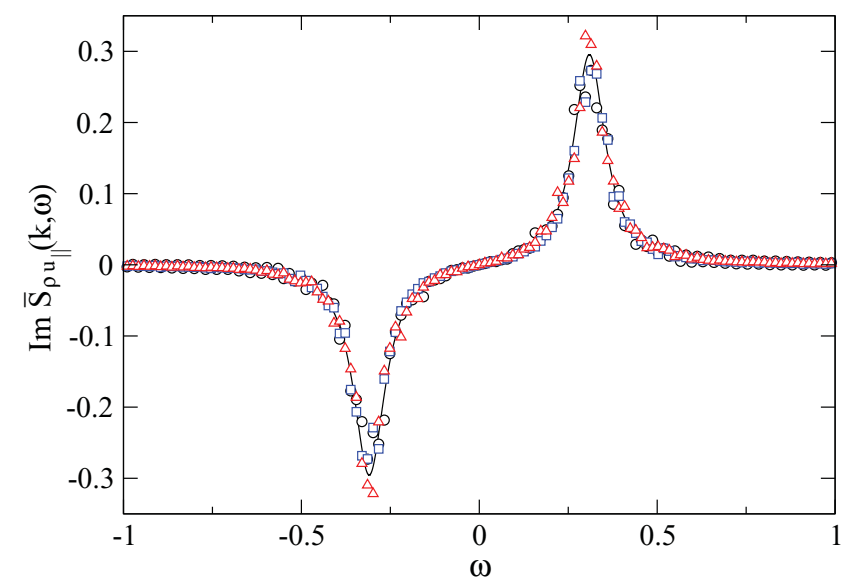

FIG. 3. (Color online) Density-velocity correlation function of a MPCD fluid. Continuous line corresponds to the prediction obtained from thermostatted FH, Eq. (38). Symbols correspond to numerical simulations of a fluid with numerical density $M=10$, collision angle $\alpha=160^{\circ}$, for wavevectors $\vec{k}=\frac{2 \pi}{L}(1,0,0)$ (circles), $\vec{k}=\frac{2 \pi}{L}(0,1,0)$ (squares), and $\vec{k}=\frac{2 \pi}{L}(0,0,1)$ (triangles).

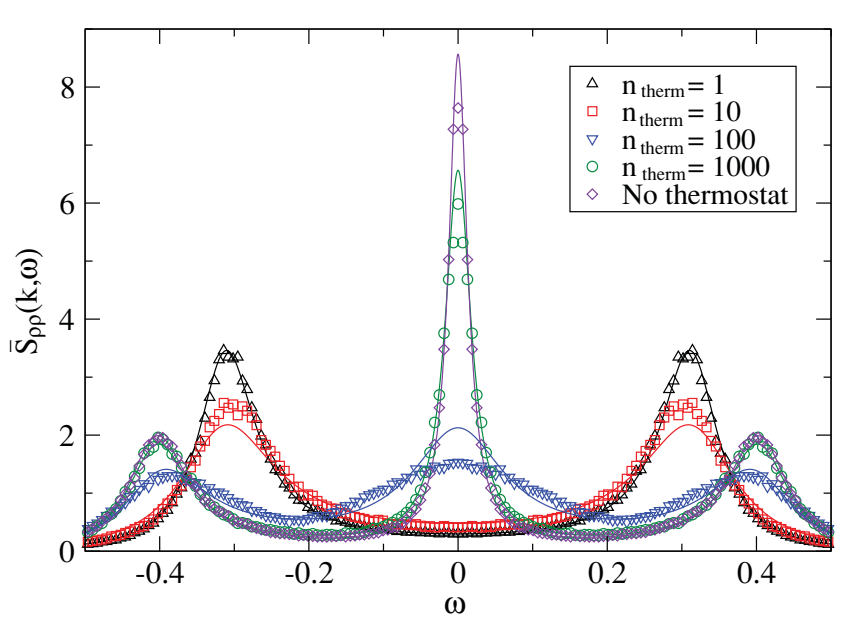

FIG. 4. (Color online) Normalized dynamic structure factor of a partially thermostatted MPCD fluid. Continuous lines represent the analytical estimation Eq. (53), with thermal diffusivities and longitudinal viscosities given by Eqs. (54)-(56) and (57)-(58), respectively. Symbols represent simulation results for numerical density $M=10$, collision angle $\alpha=130^{\circ}$, time step $\Delta t=0.1$, and different time intervals for the application of the thermostat $n_{\text {therm }} \Delta t$, with $n_{\text {therm }}=1$ (STI), 10, 100, and 1000, and no application of the thermostat. Spectra were calculated for wavevector $\vec{k}=\frac{2 \pi}{L}(1,0,0)$.

$2 \pi / L$, are considered. In this case, Eqs. (42)-(45) can be used to calculate the hydrodynamic modes of the thermostatted fluid only approximatively, and Eq. (53) shows deviations with respect to the dynamic structure factor observed in simulations, even for the isothermal and adiabatic situations. However, our model for thermostatting interactions, expressed by Eqs. (9) and (21), is still a good approximation to describe the propagation of these mesoscopic modes. This is shown in Fig. 5, where the normalized dynamic structure factor for different thermostating rates and wavenumbers $k L / 2 \pi=3,5,7$ are presented. In this figure, the fitting of the numerical results have been performed by using Eq. (40), in which the thermodynamic limit has not been applied.

\section{CONCLUSIONS}

We applied a local thermostating procedure to a fluid simulated by MPCD and analyzed the behavior of the correlation functions of hydrodynamic fluctuations in this system. By considering the characteristic timescales involved in the evolution of hydrodynamic perturbations, we suggested that the application of the thermostat at every simulation step (STI) modifies in an effective way the relaxation rate of temperature fluctuations of MPCD fluids since they are forced to decay faster than the remaining hydrodynamic modes. When the thermostat is applied at larger regular simulation-step intervals (PTI), this relaxation time is also modified but to a smaller extent.

We used FH with the purpose of studying the spectra of spontaneous fluctuations occurring under such conditions from a theoretical point of view. This is justified because correlation functions in MPCD fluids have been shown to be in agreement with linearized hydrodynamics [30], and 

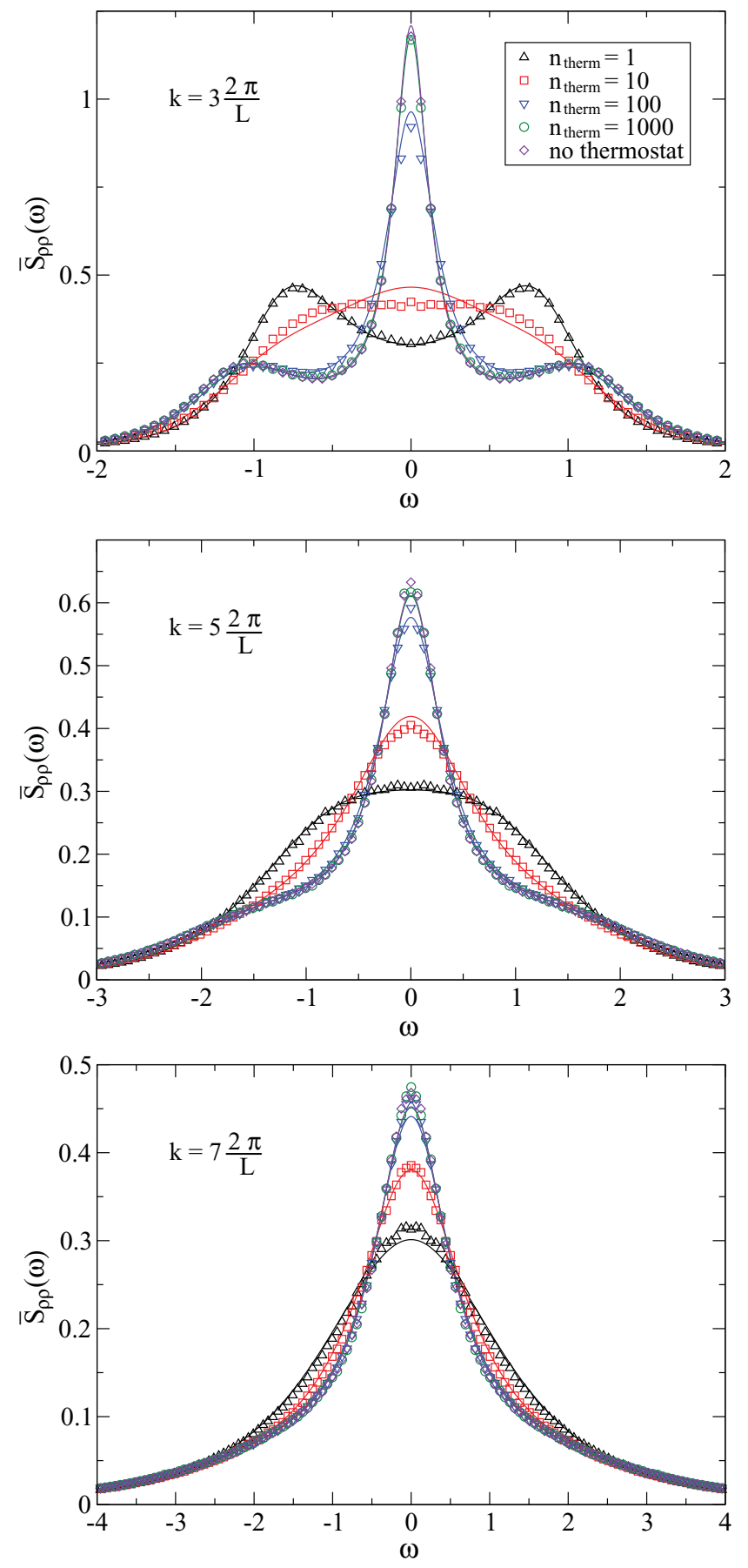

FIG. 5. (Color online) Density autocorrelation function in MPCD for different values of the wavenumber $k$. Simulation results are shown by symbols, and lines represent the theoretical fit obtained from Eq. (40).

FH can be used to describe the spatial and time evolution of hydrodynamic fluctuations in the linear regime. The main difference of our theoretical analysis with respect to the usual description of fluctuations in liquids consisted in considering the explicit interaction with a local thermostat that is applied at regular time intervals. When the thermostat is applied at every simulation step, we showed that temperature fluctuations evolve toward equilibrium on a very short timescale and can be identified as fast variables in comparison with density and velocity fluctuations that relax toward equilibrium on slower timescales. By applying a timescale separation procedure, it was then possible to obtain a reduced description in terms of the slow variables only, from which the dynamic structure factor, the autocorrelation function of the longitudinal velocity, and the density-velocity cross-correlation were obtained, Eqs. (36), (37), and (38). These correlations were compared with those obtained directly from simulations of strongly thermostatted MPCD for different densities, and the results were found to be in very good agreement.

In the general case, we solved for the hydrodynamic modes of a thermostatted fluid in terms of the time interval for the application of the thermostat and under the usual hydrodynamic approximation $c_{T} k \gg D_{l} k^{2}, D_{T} k^{2}$. We used these results to obtain the dynamic structure factor of a fluid interacting with the thermostat. In order to compare our analytical and numerical results, we have proposed that the material parameters of the fluid are not significantly modified by the thermostat. This allows for recovering the expected behavior of the dynamic structure factor in a very simple way in the whole range from no application of the thermostat to application of the thermostat at every simulation step. However, it is worth stressing that this assumption is justified only after the good agreement observed between analytical and numerical results. In principle, the rigorous justification for this approximation can be achieved from kinetic theory or projection operator techniques, which have been used elsewhere to calculate the kinetic and collisional contributions of $v$ and $D_{T}$ [18,30-32]. This calculation goes beyond the scope of this work, in which emphasis laid upon the mesoscopic behavior of thermostatted MPCD fluids. In conclusion, it is fair to say that our model can be considered as a good approximation that is useful for estimating relaxation rates of hydrodynamic fluctuations in partially thermostatted MPCD fluids.

Our results show that the application of a thermostat might change drastically the way in which fluctuations propagate in MPCD. In addition, they show that the dynamics of fluctuations under the thermostatting procedure that tends to restore the temperature on the cell level at every simulation step is the one to be expected for an isothermal liquid with an ideal equation of state, since the thermal mode is eliminated, while density and logitudinal velocity perturbations propagate at the isothermal sound speed. Finally, if partial thermostatting is implemented, e.g., in order to reduce computational costs, then it should be taken into account that propagation of the hydrodynamic modes will exhibit intermediate features between the adiabatic and the isothermal cases. Our model allows for an approximate estimation of these properties.

\section{ACKNOWLEDGMENTS}

H.H. acknowledges financial support from DAAD, Germany (2008), and DGAPA-UNAM, Mexico (2009-2010). G.S. would like to thank G. Gompper and R. Winkler for helpful discussions. 


\section{APPENDIX A: GENERATING RANDOM VARIATES FROM DISTRIBUTION EQ. (7)}

The acceptance-rejection method was invented by John von Neumann [49,50] and allows one to draw random numbers from almost arbitrary probability distribution functions. Given a distribution $p(x)$, from which random variates are to be sampled, a second distribution function $g(x)$ is chosen for which it is known how to generate random variates. Given a constant value $c$ such that $c g(x)>p(x), \forall x \in \mathbb{R}$ [the simplest choice would be $g(x)=$ const. and $c g(x)=\max p(x)]$, the algorithm proceeds in three steps: (i) choose a variate $\xi_{1} \mapsto$ $G\left(\sigma_{g}^{2}, \mu_{g}\right)$, where $G(.,$.$) is a generator of a random variate from$ the distribution $g(x)$ with variance $\sigma_{g}^{2}$ and average value $\mu_{g}$; (ii) draw a second random variate $\xi_{2} \in[0,1]$ from a uniform distribution; (iii) if $\xi_{2}<p\left(\xi_{1}\right) / \operatorname{cg}\left(\xi_{1}\right)$, accept $\xi_{1}$ as random number, otherwise repeat steps (i) to (iii). The distribution function of random variates $\xi_{1}$ will then correspond to $p(x)$.

Random energies for the distribution function $P\left(E_{\mu}\right)$, Eq. (7), were chosen by means of the acceptance-rejection method, where energies $E_{\mu} \in \mathbb{R}^{+}$are admissible. For simplicity, we will omit the subscript $\mu$ in the following discussion. The restricting function in the acceptance-rejection method was chosen as exponential distribution

$$
g(E)=\alpha \exp \left(-\alpha\left|E-E_{\mathrm{m}}\right|\right),
$$

with $E_{\mathrm{m}}=(\phi / 2-1) / \beta$ the position of the maximum, $\max [P(E)]=P\left(E_{\mathrm{m}}\right)$, and $\beta=1 / k_{B} T$.

A convenient choice for $\alpha$ was found as $\alpha=\beta \sqrt{2 / \phi}$, which ensures $g(E)>P(E), \forall E$. Figure 6 (left) shows a comparison between the probability distribution $P(E ; \beta, \phi)$ and the restricting function $g(E ; \alpha, \phi)$ with values for $\alpha$ according to this criterion. The rejection function is given as the ratio between $P(E)$ and $g(E)$, which is

$$
q_{0}(E ; \alpha, \beta, \phi)=\frac{1}{\alpha \Gamma(\phi / 2)} \beta^{\phi / 2} E^{\phi / 2-1} e^{-\beta E} e^{|E-(\phi / 2-1) / \beta| \alpha} .
$$

For the most efficient method it is useful to have the function normalized to its maximum value, i.e., $q(E ; \alpha, \beta, \phi)=$ $q_{0}(E ; \alpha, \beta, \phi) / \max \left[q_{0}(E ; \alpha, \beta, \phi)\right]$ normalized to 1 . The energy $E^{*}$ at the maximum position of $q_{0}$ is found to be $E^{*}=(\phi / 2-$ $1) /(\beta-\alpha)$. The normalized rejection function is thereby found to be

$$
q\left(E ; \alpha^{*}, \beta, \phi\right)=\frac{E^{\phi / 2-1} e^{-\beta E} e^{|\beta E-(\phi / 2-1)| \alpha^{*}}}{\left[\frac{\phi / 2-1}{\beta\left(1-\alpha^{*}\right)}\right]^{\phi / 2-1} e^{(\phi / 2-1)\left(1+\alpha^{*}\right)}},
$$

where we have introduced the temperature-scaled parameter $\alpha^{*}=\alpha / \beta$. Figure 6 (right) shows $q\left(E ; \alpha^{*}, \beta, \phi\right)$ for different numbers of degrees of freedom.

For larger values of $\phi, P(E)$ can be approximated by its limiting distribution, i.e., the shifted Gauss distribution with variance $3 k_{B} T$ and average value $E_{\mathrm{m}}$. Since the generation of Gaussian random numbers is faster than by the acceptancerejection method, $P(E)$ is approximated by a Gaussian for $\phi>100$. As shown in Fig. 7 this approximation introduces an absolute error $\epsilon<1 \%$, which seems to be tolerable for the present investigation.
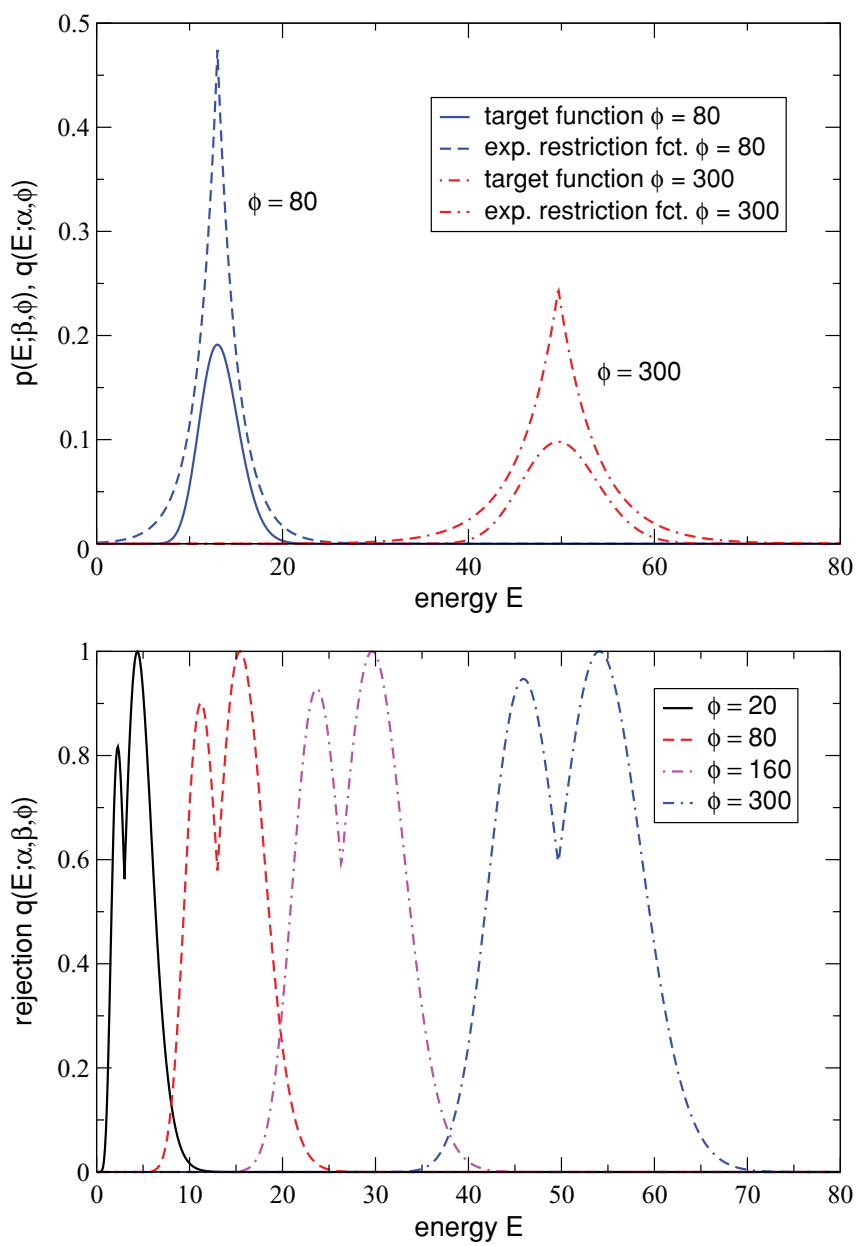

FIG. 6. (Color online) Left: comparison between restricting function and the energy distribution function, Eq. (7). Right: the normalized rejection function, Eq. (A3).

\section{APPENDIX B: CALCULATION OF $\sigma_{\text {therm }}$}

Here we will estimate the strength of the energy fluctuations induced by the thermostat $\sigma_{\text {therm }}$. With this purpose we will consider Eq. (7), which expresses the conditional probability, $P\left(E_{\mu} \mid N_{\mu}\right)$, for observing the energy $E_{\mu}$ within a MPCD cell

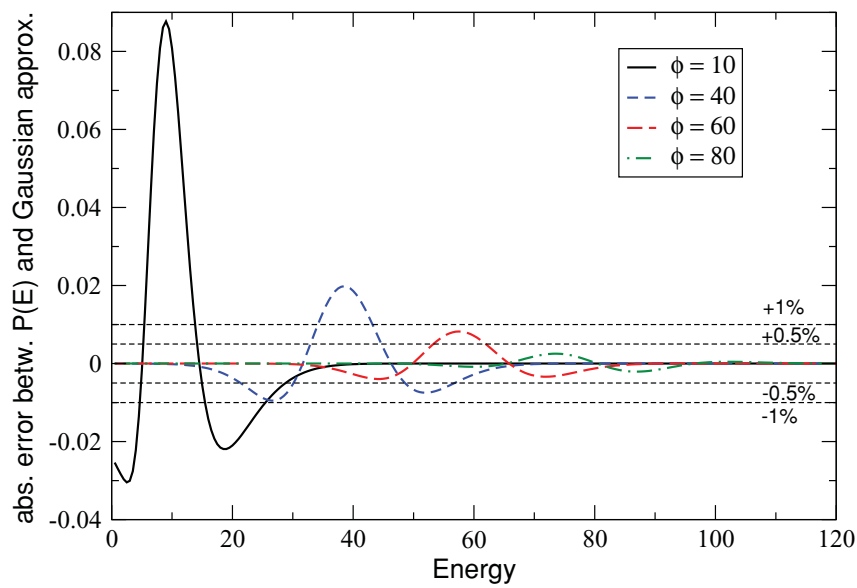

FIG. 7. (Color online) Difference between the energy distribution function, Eq. (7), and a Gaussian distribution function. 
after applying the MBST, given that the number of particles in the cell is $N_{\mu}$. Let $P\left(E_{\mu}, N_{\mu}\right)$ be the joint probability for observing a cell with energy $E_{\mu}$ and number of particles $N_{\mu}$ after thermostatting, then the reduced probability for observing the energy $E_{\mu}$ regardless of the number of particles in the cell is

$$
P\left(E_{\mu}\right)=\sum_{N_{\mu}=1}^{\infty} P\left(E_{\mu}, N_{\mu}\right)=\sum_{N_{\mu}=1}^{\infty} P\left(N_{\mu}\right) P\left(E_{\mu} \mid N_{\mu}\right)
$$

where $P\left(N_{\mu}\right)$ is the probability for observing $N_{\mu}$ particles. For MPCD, it is well known that the number of particles per cell follows a Poisson distribution within a very good approximation, i.e., $P\left(N_{\mu}\right)=M^{N_{\mu}} e^{-M} / N_{\mu}$ !. However, this expression for $P\left(N_{\mu}\right)$ makes the explicit calculation of $P\left(E_{\mu}\right)$ rather complicated. In order to simplify the analysis, we will suppose that fluctuations in the number of particles per cell are small in such a way that $P\left(N_{\mu}\right) \simeq \delta_{M N_{\mu}}$ [48]. Replacing the previous expression for $P\left(N_{\mu}\right)$ and Eq. (7) into Eq. (B1), we obtain

$$
P\left(E_{\mu}\right) \simeq \frac{1}{E_{\mu} \Gamma\left[\frac{3}{2}(M-1)\right]}\left(\frac{E_{\mu}}{k_{B} T_{0}}\right)^{\frac{3}{2}(M-1)} e^{-E_{\mu} / k_{B} T_{0}} .
$$

As indicated in Sec. III A, we will further approximate $P\left(E_{\mu}\right)$ by a Gaussian distribution. As usual, this can be done by expanding the logarithm of $P\left(E_{\mu}\right)$ in a Taylor series around its maximum which is located at $\bar{E}_{\mu}=k_{B} T_{0}(3 M-5) / 2$. This procedure yields

$$
P\left(E_{\mu}\right) \simeq \frac{1}{\sqrt{2 \pi s^{2}}} \exp \left[\frac{\left(E_{\mu}-\bar{E}_{\mu}\right)^{2}}{2 s^{2}}\right],
$$

where

$$
s^{2}=\frac{1}{2} k_{B}^{2} T_{0}^{2}(3 M-5)
$$

is the variance of the approximated distribution.

We can use Eq. (B4) in order to approximate the correlation function for the noise term $\delta \mathcal{E} \propto E(\vec{r}, t)-E_{0} \simeq E_{\mu}-\bar{E}_{\mu}$, which is the local fluctuation of energy per unit volume induced by the thermostat. It can be noticed that since the thermostat is applied only at regular time intervals we have

$$
\begin{aligned}
& \left\langle\delta \mathcal{E}(\vec{r}, t) \delta \mathcal{E}\left(\vec{r}^{\prime}, t^{\prime}\right)\right\rangle \\
& \quad= \begin{cases}\frac{1}{2 a^{6}} k_{B}^{2} T_{0}^{2}(3 M-5), & \text { if } \vec{r}=\vec{r}^{\prime} \text { and } t=t^{\prime}=q \tau \\
0, & \text { otherwise }\end{cases}
\end{aligned}
$$

where $q$ is an integer number. This expression indicates that $\delta \mathcal{E}$ is neither correlated for different cells neither for different times. In addition, the correlation vanishes whenever the thermostat is not applied.

In MPCD simulations, both space and time are discrete. Fields are measured at the cell level and at times that have the form $t=n \Delta t, t^{\prime}=n^{\prime} \Delta t$. For large systems and long simulation times, Eq. (B5) can be recast into the continuous form

$$
\left\langle\delta \mathcal{E}(\vec{r}, t) \delta \mathcal{E}\left(\vec{r}^{\prime}, t^{\prime}\right)\right\rangle=\frac{\tau}{2 a^{3}} k_{B}^{2} T_{0}^{2}(3 M-5) \delta\left(\vec{r}-\vec{r}^{\prime}\right) \delta\left(t-t^{\prime}\right),
$$

from which $\sigma_{\text {therm }}^{2}$ is identified to be

$$
\sigma_{\text {therm }}^{2}=\frac{\tau}{2 a^{3}} k_{B}^{2} T_{0}^{2}(3 M-5) .
$$

\section{APPENDIX C: TIMESCALE SEPARATION}

We will consider here the application of the timescale separation formalism for linear systems of Geigenmüller, Titulaer, and Felderhof $[45,46]$ to a strongly thermostatted system described by Eq. (23). In this formalism a system of slow, $\vec{x}$, and fast, $\vec{y}$, variables with the same dimensions coupled by the linear equation

$$
\partial_{t}\left(\begin{array}{l}
\vec{x} \\
\vec{y}
\end{array}\right)=-\left(\begin{array}{ll}
\mathbf{M}_{x \boldsymbol{x}} & \mathbf{M}_{\boldsymbol{x} \boldsymbol{y}} \\
\mathbf{M}_{\boldsymbol{y} \boldsymbol{x}} & \mathbf{M}_{\boldsymbol{y} \boldsymbol{y}}
\end{array}\right)\left(\begin{array}{l}
\vec{x} \\
\vec{y}
\end{array}\right)
$$

is considered.

The distinction between slow and fast variables emerges from the observation that $\mathbf{M}_{x x} \sim \tau_{s}^{-1}$, while $\mathbf{M}_{y y} \sim \tau_{f}^{-1}$, with $\varepsilon=\tau_{f} / \tau_{s} \ll 1$. Here we shall assume that Eq. (C1) can be recast directly in the form

$$
\partial_{t}\left(\begin{array}{l}
\vec{x} \\
\vec{y}
\end{array}\right)=-\left[\left(\begin{array}{ll}
0 & 0 \\
0 & \mathbf{F}
\end{array}\right)+\left(\begin{array}{ll}
\mathbf{A} & \mathbf{B} \\
\mathbf{C} & \mathbf{D}
\end{array}\right)\right]\left(\begin{array}{l}
\vec{x} \\
\vec{y}
\end{array}\right),
$$

where the matrix $\mathbf{F}$ is order $\tau_{f}^{-1}$, and $\mathbf{A}, \mathbf{B}, \mathbf{C}$, and $\mathbf{D}$ are all order $\tau_{s}^{-1}$. It should be stressed that the general timescale separation procedure of Refs. [45,46] considers cases where a strong coupling between $\vec{x}$ and $\vec{y}$ exists, for instance, because $\mathbf{M}_{x y} \sim \tau_{f}^{-1}$. In these cases a linear change of variables from $\vec{x}$ to $\vec{x}{ }^{\prime}=\vec{x}+\mathbf{C}_{0} \vec{y}$ yields also an equation of the form Eq. (C2). From Eq. (C2) it has been shown in Refs. [45,46], that on the slow timescale, i.e., for $t \gg \tau_{f}$, the dynamics of $\vec{x}$ can be rigorously approximated by a reduced equation of the form

$$
\partial_{t} \vec{x}=-\mathbf{M}_{x x}^{\mathrm{red}} \vec{x}
$$

which does not involve the fast variables $\vec{y}$. The reduced matrix, $\mathbf{M}_{x x}^{\text {red }}$, can be written as an expansion in powers of $\varepsilon[45,46]$,

$$
\begin{aligned}
\mathbf{M}_{x x}^{\mathrm{red}}= & \mathbf{A}-\varepsilon \mathbf{B} \mathbf{F}^{-1} \mathbf{D} \\
& +\varepsilon^{2}\left(\mathbf{B} \mathbf{F}^{-1} \mathbf{D} \mathbf{F}^{-1} \mathbf{C}-\mathbf{B F}^{-2} \mathbf{C A}\right)+\mathcal{O}\left(\varepsilon^{3}\right) .
\end{aligned}
$$

It can be easily shown that Eq. (22) can in fact be written in the form of Eq. (C2), by choosing

$$
\mathbf{A}=\left(\begin{array}{cc}
0 & c_{T} k \\
-c_{T} k & D_{l} k^{2}
\end{array}\right), \quad \mathbf{B}=\left(\begin{array}{c}
0 \\
-c_{T} k
\end{array}\right)
$$

$\mathbf{C}=\left(0,(\gamma-1) c_{T} k\right), \mathbf{D}=0, \mathbf{F}=\gamma D_{T} k^{2}+\tau^{-1}$. Explicit calculation of the products appearing on the right-hand side of Eq. (C4) with the previous expressions for A ...F, yields Eq. (23). 


\section{APPENDIX D:THEMOSTATTED HYDRODYNAMIC MODES}

The hydrodynamic modes of the fluid described by Eq. (17) are given by the roots of $\operatorname{det} \mathbf{M}(\vec{k}, \omega)=0$. The explicit form of the previous expression is

$$
\begin{aligned}
& z\left(z+D_{l} k^{2}\right)\left(z+\gamma D_{T} k^{2}+\tau^{-1}\right)+z \gamma c_{T}^{2} k^{2} \\
& \quad+\gamma c_{T}^{2} k^{2}\left(D_{T} k^{2}+\tau^{-1}\right)=0,
\end{aligned}
$$

where we have defined $z=-i \omega$, for simplicity. We will find the solutions of Eq. (D1) under the approximation $c_{T} k \gg$ $D_{l} k^{2}, D_{T} k^{2}$, while $\tau$ will be an unrestricted quantity. Up to zero order in the small quantities Eq. (D1) reads

$$
z^{(0) 3}+z^{(0) 2} \tau^{-1}+z^{(0)} \gamma c_{T}^{2} k^{2}+c_{T}^{2} k^{2} \tau^{-1}=0,
$$

which has three roots given by Eqs. (42)-(44) with

$$
\begin{gathered}
B=\left(P+\sqrt{P^{2}+Q^{3}}\right)^{\frac{1}{3}}+\left(P-\sqrt{P^{2}+Q^{3}}\right)^{\frac{1}{3}}, \\
A=\left(P+\sqrt{P^{2}+Q^{3}}\right)^{\frac{1}{3}}-\left(P-\sqrt{P^{2}+Q^{3}}\right)^{\frac{1}{3}}, \\
P=\frac{1}{54}\left[9 c_{T}^{2} k^{2}(\gamma-3) \tau^{-1}-2 \tau^{-3}\right], \\
Q=\frac{1}{9}\left(3 \gamma c_{T}^{2} k^{2}-\tau^{-2}\right) .
\end{gathered}
$$

Up to first order in the small-frequency $D_{l} k^{2}$, the secular equation reduces to

$$
\begin{aligned}
& z^{(1)}\left(3 z^{(0) 2}+\gamma c_{T}^{2} k^{2}+2 z^{(0)} \tau^{-1}\right)+D_{l} k^{2}\left(z^{(0) 2}+z^{(0)} \tau^{-1}\right) \\
& \quad+\gamma D_{T} k^{2}\left(z^{(0) 2}+c_{T}^{2} k^{2}\right)=0 .
\end{aligned}
$$

By solving this expression for $z^{(1)}$, and replacing the roots of order zero, $s_{i}^{(0)}$, Eq. (45) is obtained.
[1] A. Malevanets and R. Kapral, J. Chem. Phys. 110, 8605 (1999)

[2] D. Frenkel and B. Smith, Understanding Molecular Simulations: From Algorithms to Applications (Academic Press, San Diego, 2002).

[3] D. C. Rapaport, The Art of Molecular Dynamics Simulation, 2nd ed. (Cambridge University Press, Cambridge, 2005).

[4] G. Sutmann, in Multiscale Simulations Methods in Molecular Sciences, edited by J. Grotendorst, N. Attig, S. Blügel, and D. Marx, Vol. 42 (John von Neumann Institute for Computing, Jülich, 2009), p. 1.

[5] D. L. Ermak and Y. Yeh, Chem. Phys. Lett. 24, 243 (1974).

[6] D. L. Ermak, J. Chem. Phys. 62, 4189 (1975).

[7] J. F. Bradly and G. Bossis, Annu. Rev. Fluid Mech. 20, 111 (1988).

[8] U. Frisch, B. Hasslacher, and Y. Pomeau, Phys. Rev. Lett. 56, 1505 (1986).

[9] S. Chen and G. D. Doolen, Ann. Rev. Fluid Mech. 30, 329 (1998).

[10] S. Succi, The Lattice Boltzmann Equation for Fluid Dynamics and Beyond (Oxford University Press, Oxford, 2001).

[11] A. Malevanets and R. Kapral, J. Chem. Phys. 112, 7260 (2000).

[12] S. H. Lee and R. Kapral, J. Chem. Phys. 121, 11163 (2004).

[13] N. Kikuchi, J. F. Ryder, C. M. Pooley, and J. M. Yeomans, Phys. Rev. E 71, 061804 (2005).

[14] J. T. Padding and A. A. Louis, Phys. Rev. E 74, 031402 (2006).

[15] J. M. Yeomans, Physica A 369, 159 (2006).

[16] E. Tüzel, M. Strauss, T. Ihle, and D. M. Kroll, Phys. Rev. E 68, 036701 (2003).

[17] T. Ihle and D. M. Kroll, Phys. Rev. E 63, 020201 (2001).

[18] T. Ihle and D. M. Kroll, Phys. Rev. E 67, 066706 (2003).

[19] C. M. Pooley and J. M. Yeomans, J. Phys. Chem. B 109, 6505 (2005).

[20] N. Kikuchi, A. Gent, and J. M. Yeomans, Eur. Phys. J. E 9, 63 (2002).

[21] A. Malevanets and J. M. Yeomans, Europhys. Lett. 52, 231 (2000).

[22] M. Ripoll, K. Mussawisade, R. G. Winkler, and G. Gompper, Europhys. Lett. 68, 106 (2004).

[23] A. Lamura, G. Gompper, T. Ihle, and D. M. Kroll, Europhys. Lett. 56, 319 (2001).

[24] E. Allahyarov and G. Gompper, Phys. Rev. E 66, 036702 (2002).
[25] H. Noguchi and G. Gompper, Phys. Rev. E 72, 011901 (2005).

[26] N. Kikuchi, C. M. Pooley, J. F. Ryder, and J. M. Yeomans, J. Chem. Phys. 119, 6388 (2003).

[27] A. W. Lees and S. F. Edwards, J. Phys. C 5, 1921 (1972).

[28] M. Hecht, J. Harting, T. Ihle, and H. J. Herrmann, Phys. Rev. E 72, 011408 (2005).

[29] L. D. Landau and E. M. Lifshitz, Fluid Mechanics, 2nd rev. English ed. (Pergamon, London, 1959)

[30] E. Tüzel, T. Ihle, and D. M. Kroll, Phys. Rev. E 74, 056702 (2006).

[31] T. Ihle, E. Tüzel, and D. M. Kroll, Phys. Rev. E 70, 035701 (2004).

[32] T. Ihle, E. Tüzel, and D. M. Kroll, Phys. Rev. E 72, 046707 (2005).

[33] R. G. Winkler, in Computational Condensed Matter Physics, Lecture Notes of 37th IFF Spring School, edited by J. K. G. Dhont, G. Gompper, and D. Richter (Research Center, Jülich, 2008).

[34] G. Gompper, T. Ihle, D. M. Kroll, and R. G. Winkler, in Advances in Polymer Science, edited by C. Holm and K. Kremer, Vol. 221 (Springer, Berlin, 2009), p. 1.

[35] D. M. Heyes, Chem. Phys. 82, 285 (1983).

[36] C. C. Huang, A. Chatterij, G. Sutmann, G. Gompper, and R. G. Winkler, J. Comput. Phys. 229, 168 (2010).

[37] D. Forster, Hydrodynamic Fluctuations, Broken Symmetry and Correlation Functions (W. A. Benjamin, Reading, 1975).

[38] B. J. Berne and R. Pecora, Dynamic Light Scattering: With Applications to Chemistry, Biology, and Physics (Dover, New York, 2000).

[39] J. M. Ortiz de Zárate and J. V. Sengers, Hydrodynamic Fluctuations in Fluids and Fluid Mixtures (Elsevier, Amsterdam, 2006).

[40] H. Híjar and R. F. Rodríguez, Phys. Rev. E 69, 051701 (2004).

[41] J. F. Camacho, H. Híjar, and R. F. Rodríguez, Physica A 348, 252 (2005).

[42] R. F. Rodríguez and H. Híjar, Eur. Phys. J. B 50, 105 (2006).

[43] I. O. Götze, H. Noguchi, and G. Gompper, Phys Rev. E 76, 046705 (2007).

[44] R. F. Fox, Phys. Rep. 48, 180 (1978).

[45] U. Geigenmüller, U. M. Titulaer, and B. U. Felderhof, Physica A 119, 41 (1983). 
[46] U. Geigenmüller, U. M. Titulaer, and B. U. Felderhof, Physica A 119, 53 (1983).

[47] An extension to MPC exists, which conserves angular momentum [43]. It is not based on the rotation principle (as it is for SRD), but velocities of all particles in a collision cell are drawn randomly from a Gaussian distribution under the condition to conserve momentum, energy, and angular momentum.

[48] This approximation is introduced mainly for mathematical convenience but could be questionable because the distribution of particles in MPCD cells does not vanish for $N_{\mu} \neq M$. Instead, $P\left(N_{\mu}\right)$ is rather flat in the vicinity of $M$. A correct procedure would consist in performing a saddle-point expansion of the distribution $P\left(N_{\mu}\right)$ around $N_{\mu}=M$ and evaluating the resulting sum. However, a detailed analysis shows that this approximation yields results that are very close to those presented in this paper.

[49] J. von Neumann, Nat. Bureau Standards 12, 36 (1951).

[50] P. K. Keown, Stochastic Simulation in Physics (Springer, Singapore, 1997). 